\title{
Pflegeverläufe im Spiegel von Routinedaten der GKV: Eine Analyse für die Jahre 2003 bis 2010
}
Christian Hagist
Daniel Ehing
Tobias Saal
WHU - Otto Beisheim
Universität Freiburg
School of Management
Forschungszentrum
Generationenverträge
christian.hagist@whu.edu daniel.ehing@vwl.uni-freiburg.de

WHU - Working Paper

Series in Economics

WP 14/01 


\title{
Pflegeverläufe im Spiegel von Routinedaten der GKV: Eine Analyse für die Jahre 2003 bis 2010
}

\author{
Daniel Ehing, Christian Hagist, Tobias Saal*
}

September 2014

\section{Zusammenfassung}

Die vorliegende Arbeit befasst sich auf Basis von Routinedaten der AOK-Plus mit Pflegeverläufen von über 60Jährigen in Deutschland. Unsere Analysen zeigen eine rechtsschiefe Verteilung der Pflegeverläufe für beide Geschlechter. Demnach sind 50 Prozent der weiblichen (männlichen) Pflegefälle nach 45 (26) Monaten verstorben, wohingegen die mittlere Verweildauer in der Pflege bei 51,4 (36,6) Monaten liegt. Die mit diesen Pflegverläufen verbundenen Gesamtkosten der Pflege summieren sich nach unseren Berechnungen im Mittel auf einen Barwert von 68.871 (45.233) Euro. Die Analyse der Pflegestufen mittels eines multinominalen logistischen Modells zeigt für die vorliegenden Daten zudem, dass im Beobachtungszeitraum eine Kompression der Pflegeverläufe stattgefunden hat. So wird eine höhere Pflegestufe und somit ein schwerwiegenderer Pflegeverlauf mit zunehmendem Abstand vom Tod sowie zunehmendem Alter für beide Geschlechter unwahrscheinlicher.

Stichwörter: Pflegeverlauf, Kosten der Pflege, Kompression des Pflegeverlaufs

\footnotetext{
* Kontakt: daniel.ehing@vwl.uni-freiburg.de, Forschungszentrum Generationenverträge, Albert-Ludwigs-Universität Freiburg. Teile der vorliegenden Arbeit entstanden im Rahmen der Diplomarbeit von Herrn Tobias Saal (2012). Ein besonderer Dank für die Bereitstellung der Daten geht an die AOK-Plus und dabei insbesondere an Frau Ines Krause. Für hilfreiche Hinweise danken wir ebenfalls Natalie Laub, Stefan Moog und Christoph Metzger. Für alle verbleibenden Fehler zeichnen die Autoren verantwortlich.
} 


\section{Einleitung}

Diskussionen über die zukünftige Ausgestaltung der Pflegeversicherung finden oftmals im Spannungsfeld zwischen gewünschter Leistungsausweitung sowie der damit induzierten Ausgaben- und somit Beitragssatzsteigerung statt. Der Ausbau der Pflegeversicherung wird dabei von der einen Seite als zu zögerlich empfunden, wohingegen andere Beteiligte den Deckungsgrad der Pflegeversicherung als ausreichend (bzw. bereits zu hoch) erachten, und die Abdeckung von weiteren Leistungen über eine wie auch immer organisierte private Vorsorge fordern. In allen Diskussionen unbestritten ist dabei, dass in Zukunft mit einer steigenden Zahl an Pflegefällen zu rechnen ist: Je nach Projektionsszenario wird sich aufgrund der Altersverschiebung in der Bevölkerung ein Anstieg der Pflegefälle von rund 2,3 Millionen heute auf 3,2 bis 5,9 Millionen im Jahr 2050 ergeben. ${ }^{1}$

Neben der zukünftigen Zahl der Pflegefälle und dem Leistungsgrad der Pflegeversicherung wird jedoch auch der zukünftige Pflegeverlauf die Ausgaben der Pflegeversicherung mit bestimmen. Eine fundierte Diskussion der zukünftigen Absicherung des Pflegerisikos sollte daher auch diesen Baustein mitbetrachten, zumal sich nur dann abschätzen lässt, wie sich eine Neufassung des Pflegebegriffs auswirken könnte und welche Auswirkungen von einer weiter steigenden Lebenserwartung auf den Pflegeverlauf zu erwarten sind. Aufgrund der eingeschränkten Verfügbarkeit von Längsschnittdaten für die Pflege ist die bisherige Zahl der Studien, die sich mit dem genauen Pflegeverlauf befassen, jedoch gering. Vorliegende Arbeit erweitert daher den Forschungsstand um eine empirische Analyse des Pflegeverlaufs. Hierbei wird nicht nur herausgearbeitet, wie sich die Unterschiede nach dem Alter, dem Geschlecht und der Ersteinstufung in die Pflege darstellen, sondern es wird darüber hinaus auch mittels eines logistischen Modells gezeigt, wie sich ein Anstieg der Lebenserwartung in Zukunft auf die Verweildauer in der Pflege auswirken könnte. Die vorliegende Studie kann daher als Ausgangsbasis für eine Prognose der (zukünftigen) Pflegekosten gesehen werden.

Die Arbeit ist wie folgt aufgebaut: Abschnitt 2 gibt zunächst einen kurzen Einstieg in das System der deutschen Pflegeversicherung und fasst die bisherigen Erkenntnisse zum Pflegeverlauf zusammen. Darauf folgend wird in Abschnitt 3 die Datenbasis vorgestellt und die Verweildauer in der Pflege analysiert, wobei ein besonderes Augenmerk auf die Pflegekosten gelegt wird. Abschnitt 4 versucht im Anschluss mittels eines multinominalen logistischen Modells die

\footnotetext{
${ }^{1}$ Vgl. Blinkert und Klie (2001)
} 
Auswirkungen einer steigenden Lebenserwartung auf den Pflegeverlauf abzuschätzen. Am Ende werden in einem Fazit die Ergebnisse zusammengefasst.

\section{Die soziale Pflegeversicherung: eine kurze Einführung}

Die soziale Pflegeversicherung (SPV) wurde am 1. Januar 1995 als Teilkaskoabsicherung des Pflegerisikos unter dem Dach der gesetzlichen Pflegeversicherung etabliert und ist heute, am Finanzvolumen gemessen (2012: 21,9 Milliarden Euro), nach der Unfallversicherung die zweitkleinste der fünf Sozialversicherungssäulen. ${ }^{2}$ Durch die Koppelung der Versicherungspflicht an die gesetzliche Krankenversicherung deckt die SPV dabei rund 90 Prozent der Bevölkerung mit einem Versicherungsschutz ab, wobei ihre Finanzierung im Umlageverfahren erfolgt. Der Beitragssatz auf das versicherungspflichtige Einkommen beträgt hierbei aktuell 2,05 (kinderlos: 2,3) Prozent für die Beitragszahler.

Generell wird der Versicherungsfall Pflegebedürftigkeit vom Medizinischen Dienst der Krankenversicherung (MDK) festgestellt, wobei Pflegepatienten aufgrund des zeitlichen Hilfsbedarfs für die Grundpflege sowie des Hilfsbedarfs für die hauswirtschaftliche Versorgung in verschiedene Pflegestufen eingestuft werden. Die Pflegebedürftigkeit wird dabei je nach Schwere des Pflegefalls in drei Stufen differenziert, ${ }^{3}$ wobei die jeweilige Pflegestufe noch einmal nach der Leistungsart ambulantes Pflegegeld, ambulante Pflegesachleistung und stationäre Pflegesachleistung unterteilt wird. ${ }^{4}$ Generell steht es den Versicherten frei, zwischen ambulanten und stationären Leistung zu wählen.

Wie sich in der Vergangenheit gezeigt hat, fanden vor allem Demenzkranke nur unzulänglich Berücksichtigung in den drei Pflegestufen. Mit dem Inkrafttreten des Pflegeweiterentwicklungsgesetztes am 1. Juli 2008 wurde der Leistungskatalog daher mit der Pflegestufe 0 sowie zusätzlichen Leistungen in jeder Pflegestufe im Falle eines erheblichen Betreuungsbedarfs erweitert. Zusätzlich wurde zum 1. Juli 2008 eine Dynamisierung der seit Einführung der SPV nicht angepassten Pflegeleistungen beschlossen, die mit dem Jahr 2012 ausgelaufen ist. In-

\footnotetext{
${ }^{2}$ Für eine ausführliche Darstellung der sozialen Pflegeversicherung sei auf das BMG (2011) verwiesen.

${ }^{3}$ Pflegestufe I: erhebliche Pflegebedürftigkeit mit einem Zeitaufwand von mindestens 90 Minuten täglich; Pflegestufe II: schwere Pflegebedürftigkeit mit einem Zeitaufwand von mindestens 180 Minuten täglich; Pflegestufe III: schwerste Pflegebedürftigkeit mit einem Zeitaufwand von mindestens 300 Minuten täglich.

${ }^{4}$ Die Leistungen der ambulanten Pflege kann dabei noch weiter in Kombinationsfälle aus Pflegegeld und Pflegesachleistung sowie die teilstationäre Pflege untergliedert werden. Da diese Kategorien in vorliegender Studie jedoch keine Berücksichtigung finden, wird auf sie nicht weiter eingegangen.
} 
wieweit die Leistungen der Pflegeversicherung in Zukunft dynamisiert werden, wird diskretionär von der Politik bestimmt, wobei alle drei Jahre eine Überprüfung der Leistungen stattfinden soll.

Neben den genannten Einflussfaktoren, die sich unter den Stichwörtern „Leistungsdynamisierung“, „Ausbau des Leistungskatalogs“ sowie „Anstieg der zukünftigen Zahl der Leistungsbezieher" zusammenfassen lassen, wird das langfristige Ausgabevolumen der SPV auch davon bestimmt, wie lange die Pflegepatienten in der Pflege verweilen und welche Pflegestufen hierbei maßgeblich nachgefragt werden. Verschiedene Studien haben sich daher bereits mit der Thematik der Pflegeverläufe auseinandergesetzt (vgl. Tabelle 1). Wie sich hierbei zeigt, unterscheiden sich die vorliegenden Arbeiten vor allem in Bezug auf die Zahl der betrachten Pflegefälle. Aber auch im Hinblick auf die Beobachtungsdauer und den berücksichtigten Altersbereich ergeben sich Unterschiede. Der uns vorliegende Datensatz sticht dabei vor allem durch die hohe Zahl der analysierten Pflegefälle heraus. Mit rund 222.000 Pflegefällen übertrifft er die Anzahl der anderen Auswertungen deutlich.

Tabelle 1: Übersicht Vergleichsstudien

\begin{tabular}{|c|c|c|c|c|c|c|}
\hline & $\begin{array}{l}\text { Eigener Daten- } \\
\text { satz }\end{array}$ & $\begin{array}{l}\text { Rothgang et al. } \\
\text { (2011) }\end{array}$ & $\begin{array}{c}\text { Häcker und } \\
\text { Hackmann } \\
\text { (2012) }\end{array}$ & $\begin{array}{l}\text { Seeger et al. } \\
\text { (2008; 2011) }\end{array}$ & $\begin{array}{c}\text { Prüß et al. } \\
(2006)\end{array}$ & $\begin{array}{c}\text { Borchert und } \\
\text { Rothgang } \\
\text { (2006) }\end{array}$ \\
\hline Pflegekasse & AOK-Plus & BARMER GEK & AOK Berlin & Deutsche BKK & AOK Hessen & BARMER GEK \\
\hline Pflegefälle & 221.991 & 24.602 & 114.403 & 88.575 & 6.298 & 17.821 \\
\hline Altersbereich & $60+$ & komplett & komplett & komplett & komplett & $50+$ \\
\hline $\begin{array}{l}\text { Beobachtungs- } \\
\text { dauer }\end{array}$ & $\begin{array}{c}8 \text { Jahre (2003- } \\
\text { 2010) }\end{array}$ & $\begin{array}{c}8 \text { Jahre (1999- } \\
\text { 2006) }\end{array}$ & $\begin{array}{l}9 \text { Jahre (2000- } \\
\text { 2009) }\end{array}$ & $\begin{array}{c}13 \text { Jahre (1995- } \\
\text { 2007) }\end{array}$ & $\begin{array}{c}5 \text { Jahre (1998- } \\
\text { 2002) }\end{array}$ & $\begin{array}{c}7 \text { Jahre (1998- } \\
\text { 2004) }\end{array}$ \\
\hline
\end{tabular}

Quelle: eigene Darstellung

Betrachtet man die Ergebnisse aller Studien in der Zusammenfassung, so lässt sich festhalten, dass eine signifikant höhere Überlebenswahrscheinlichkeit in der Pflege bei den Frauen zu beobachten ist, wobei diese für beide Geschlechter mit der Höhe der Pflegestufe bei Ersteinstufung sowie zunehmenden Alter absinkt. ${ }^{5}$ Inwiefern sich diese Beobachtungen auch für unseren Datensatz bestätigen, soll im Folgenden aufgezeigt werden.

\footnotetext{
${ }^{5}$ Vgl. Seger et al. (2008; 2011), Rothgang et al. (2013) und Häcker und Hackmann (2012).
} 


\section{Deskriptive Statistik}

\subsection{Datensatz und Datenaufbereitung}

Die vorliegenden Auswertungen basieren auf personenbezogenen Routinedaten der gesetzlichen Kranken- und Pflegekasse AOK-Plus. ${ }^{6}$ Den Autoren liegen dabei sowohl soziodemografische als auch medizinische Informationen über die aktuelle Pflegestufe sowie den aus Pflegeund Krankenversicherung verbundenen Ausgaben vor. Relevant für vorliegende Auswertungen sind die Leistungen der Pflegekasse, die sich im Datensatz nach der Pflegestufe I bis III (unterteilt in die Leistungen: Pflegegeld, Pflegesachleistung, ambulante Kombinationsfälle sowie vollstationäre Pflege), dem Status einer Pflegeunterbrechung sowie dem Zustand „Tod“ differenzieren lassen.

Für die Auswertungen des Pflegeverlaufs steht prinzipiell ein Beobachtungszeitraum vom 1. Januar 2000 bis zum 31. Dezember 2010 zur Verfügung, in welchem die Leistungsdaten tagesgenau erfasst werden. Der Datensatz ist dabei so aufgebaut, dass zum Auslesestichtag (22.07.2011) rund 1,2 Millionen Versicherte berücksichtigt werden, die am 1. Januar 200060 Jahre und älter waren. Die evtl. vorhandenen Leistungen der Pflegekasse für diese 1,2 Millionen Versicherten werden über den Beobachtungszeitraum im Datensatz dokumentiert, so dass im Januar 2010 alle beobachteten Personen 70 Jahre und älter sind.

Um eine Verzerrung durch Pflegefälle zu verhindern, die bereits vor dem 01. Januar 2000 pflegebedürftig waren, sowie um eine von der AOK-Plus bereits teilweise durchgeführte Datenarchivierung zu berücksichtigen, müssen die Daten zunächst linkszensiert werden. In Rücksprache mit den Sachverständigen der AOK-Plus wird dabei ein Zeitraum von drei Jahren gewählt, wobei dieser große Zeitraum vor allem durch eine unsystematische Datenmigration seitens des Datenlieferanten bedingt ist. Diese Vorgehensweise soll sicherstellen, dass nur neu eingetretene Pflegefälle Eingang in die Untersuchung finden. Als auswertungsrelevanter Zeitraum verbleibt somit der 1. Januar 2003 bis 31. Dezember 2010, was einer Zeitspanne von acht Jahren entspricht. Ferner liegen im Datensatz Fälle von Rechtszensur vor. Diese können zum einen dadurch bedingt sein, dass ein Versicherter die Krankenkasse wechselt oder zum anderen dadurch, dass das Ende des Beobachtungszeitraums erreicht ist und es entweder nicht zum

\footnotetext{
${ }^{6}$ Die AOK-Plus ist eine gesetzliche Krankenversicherung mit Hauptsitz in Dresden. Sie betreut ca. 2,7 Millionen Versicherte, die hauptsächlich in Sachsen und Thüringen wohnen. Für weitere Informationen vgl. www.aokplusonline.de .
} 
Eintritt des Versicherungsfalls Pflege kam oder aber ein Pflegezustand besteht, dessen weiterer Verlauf unklar ist.

Über die Links- und Rechtszensur hinaus müssen die Daten weiter aufbereitet werden, um einerseits das Problem von vorhandenen Leistungsunterbrechungen handhaben zu können und andererseits vorhandene Leistungsüberschneidungen einzelner Leistungsarten der Pflegekasse abzugrenzen. Pflegelücken entstehen im Datensatz entweder durch eine Leistungsunterbrechung, die auf eine Besserung des Gesundheitszustands zurückzuführen ist, oder durch eine Fehlkodierung bei der Datenerfassung. Um diese zwei Ursachen voneinander abzugrenzen, wird im Weiteren angenommen, dass alle Pflegelücken, die größer als 90 Tage sind, auf eine Besserung des Gesundheitszustands zurückgehen. ${ }^{7}$ Diese Lücken werden im Weiteren als Pflegeunterbrechung kodiert, wobei eine spätere Rückkehr in einen Pflegezustand möglich ist. Alle Pflegelücken, die kleiner oder gleich 90 Tage sind, werden in Absprache mit dem Datenlieferant geschlossen, wobei der Zustandsbeginn des auf die Pflegelücke folgenden Pflegestatus als Referenz herangezogen und somit der Pflegezustand vor der Leistungslücke verlängert wird. ${ }^{8}$ Neben dem Problem der Pflegelücken existieren bei vier Prozent der Pflegeeinträge Überschneidungen von Pflegeleistungen. Diese sind entweder auf eine genauere Spezifizierung von ambulanter Pflegeleistung zurückzuführen oder wiederum auf Kodierungsfehler seitens des Datenlieferanten. ${ }^{9}$ Zur Korrektur wird für Überschneidungsfälle, die auf Kodierungsfehler zurückzuführen sind, der Zustandsbeginn der überschneidenden Pflegstadien als Referenzpunkt herangezogen. ${ }^{10}$ Kodierungsfehler, die auf Überschneidungen bei den ambulanten Pflegeleistungen zurückgehen und die zumeist mit Zustandsbeginn und Zustandsende vollkommen in einem anderen ambulanten Pflegestatus liegen, werden durch den übergreifenden Pflegezustand überschrieben. Da die ambulanten Pflegeleistungen im Folgenden nicht aufgetrennt nach den einzelnen Leistungsarten betrachtet werden und die Pflegestufe sich in diesen Fällen nicht ändert, dürfte dies zu keiner Verzerrung der Ergebnisse führen.

\footnotetext{
${ }^{7}$ Für ca. drei Prozent der Zustandseinträge besteht eine nicht kodierte Pflegelücke von mehr als 90 Tagen.

${ }^{8}$ Bei ca. 30 Prozent der Zustandseinträge ist dies der Fall. Der Mittelwert (Standardabweichung) der Lücken zwischen den Einträgen beträgt 6 Tage (11 Tage).

${ }^{9}$ So liegen bspw. Kombinationsfälle aus ambulanter Pflegesachleistung und Pflegegeld oft vollkommen in dem Leistungszeitraum einer ambulanten Sachleitung.

${ }^{10}$ Insgesamt beträgt der Anteil der Zustandseinträge, die sich überschneiden und für die kein plausibler Grund für die Überschneidung gefunden werden kann, 1,5 Prozent an allen Einträgen. Die Verzerrungen, die von dieser Korrektur ausgehen, sind daher nach der Meinung der Autoren als gering einzustufen.
} 
Nach der beschriebenen Korrektur der Daten verbleiben 221.991 Pflegeverläufe, die ihren Beginn zwischen Januar 2003 und Dezember 2010 haben. Dabei sind von dieser Anzahl 150.099 (71.892) Frauen (Männer). Die Altersstruktur dieser Pflegefälle bei Pflegebeginn ist in Abbildung 4 im Anhang dargestellt. Das durchschnittliche (median) Eintrittsalter der Frauen in die Pflege beträgt 81,8 (82,2) Jahre, wohingegen das der Männer bei 78,8 (79) Jahre liegt. ${ }^{11}$ Das durchschnittliche Eintrittsalter in die Pflege liegt dabei für die Frauen (Männer) $3(6,6)$ Jahre über dem Eintrittsalter, welches der Medizinische Dienst der Krankenkassen (vgl. MDK (2002)) ermittelt, und dürfte hauptsächlich auf die Beschränkung der Daten auf alle über 60-Jährigen zurückzuführen sein. Der gleiche Grund dürfte auch dazu führen, dass beim MDK (2003) insgesamt nur 62,7 Prozent der Neueinstufungen weiblich sind, wohingegen dies in vorliegendem Datensatz bei 67,6 Prozent der Fall ist. Wie Tabelle 9 im Anhang zeigt, liegt die Ersteinstufung der Pflegefälle in die einzelnen Pflegestufen für das Jahr 2003 trotz der beschriebenen Abweichungen insgesamt betrachtet auf einem ähnlichen Niveau, so dass von einer hinreichenden Qualität der Stichprobe auszugehen ist, um den Pflegeverlauf im Weiteren aufgetrennt nach Alter und Geschlecht zu beschreiben. ${ }^{12}$ Um der Korrektur der Daten (insbesondere der Pflegelücken) gerecht zu werden, wird dabei zwar eine Auswertung auf Tagesbasis vorgenommen, jedoch werden die Ergebnisse im Weiteren nur in Monatsabständen dokumentiert.

\subsection{Verweildauern in der Pflege}

Die Auswertung der Daten beschränkt sich im Folgenden auf die Pflegestufe I bis III im ambulanten und stationären Bereich sowie den Zustand einer Pflegeunterbrechung oder den Zustand "Tod", sodass insgesamt acht Zustände möglich sind. Dem Beispiel von Häcker und Hackmann (2012) sowie Seger et al. (2008) folgend, wird für die Modellierung des Pflegeverlaufs eine Kaplan-Meier-Schätzung verwendet. ${ }^{13}$ Die resultierende Überlebensfunktion wird anschließend prozentual auf die Anteile der einzelnen Pflegestufen zu jedem Zeitpunkt aufgegliedert, was Aussagen über die Veränderung des Pflegearrangements sowie über Veränderungen der Pflegestufe ermöglicht. ${ }^{14}$ Die Auswertung der Pflegedaten erfolgt dabei aufgetrennt nach Geschlecht, Alter und der Ersteinstufung in die Pflege.

\footnotetext{
${ }^{11}$ Die Standardabweichung bei den Frauen (Männer) liegt bei 6,5 $(7,1)$ Jahren.

${ }^{12}$ Wie Rothgang et al. (2011) anmerken, ist aufgrund der Zahlungswirksamkeit der Routinedaten von einer hohen Datenvalidität auszugehen.

${ }^{13}$ Vgl. Cleves (2008).

${ }^{14} \mathrm{Vgl}$. Borchert und Rothgang (2006).
} 
Wie Abbildung 1 zeigt, beginnen 72 (63) Prozent der Frauen (Männer) ihren Pflegeverlauf in Pflegestufe I, wobei der Großteil hiervon ambulant gepflegt wird. Nach drei Monaten Pflege sind ca. 20 Prozent der Männer verstorben, wohingegen dies nur bei 11 Prozent der Frauen zutrifft. Die hohe Sterblichkeit in den Anfangsmonaten ist dabei vor allem auf jene Pflegeverläufe zurückzuführen, die mit Pflegestufe II oder III ihren Pflegeverlauf beginnen. Im weiteren zeitlichen Verlauf flacht der Anstieg der Sterblichkeit ab, und so sind in vorliegendem Datensatz 50 Prozent der Frauen nach 45 Monaten verstorben, wohingegen dies bei den Männern bereits nach 26 Monaten der Fall ist. Mithin ändert sich nach dem Pflegebeginn auch die Zusammensetzung des Pflegearrangements: Werden bei den Frauen zu Beginn nur 17 Prozent stationär gepflegt, so sind es nach 45 Monaten bereits 36 Prozent. Bei den Männern befindet sich dieser Anteil hingegen durchweg auf einem niedrigeren Niveau. So werden zu Pflegebeginn ca. 12 Prozent stationär gepflegt und diese Quote steigt nach 26 Monaten, wenn 50 Prozent der Pflegefälle verstorben sind, auf rund 22 Prozent an.

Die unterschiedlichen stationären Pflegeanteile lassen sich unter anderem darauf zurückführen, dass beim Eintritt des Pflegezustands bei den Männern die zumeist weibliche (und noch lebende) Lebenspartnerin die Pflege zu Hause (mit) übernimmt, wohingegen die informelle Pflege bei den Frauen oft nur noch eingeschränkt von den Familien geleistet werden kann, und daher teilweise zur formellen stationären Pflege übergeht. ${ }^{15}$ Zum Ende unseres Beobachtungszeitraums nach 94 Monaten sind noch rund 19 (10) Prozent der weiblichen (männlichen) Pflegefälle am Leben. Wie zu erwarten, zeigt sich auch in den vorliegenden Daten die höhere Verweildauer der Frauen in der Pflege.

\footnotetext{
${ }^{15}$ Vgl. Schupp und Künemund (2004)
} 


\section{Abbildung 1: Pflegeverläufe nach Geschlecht}

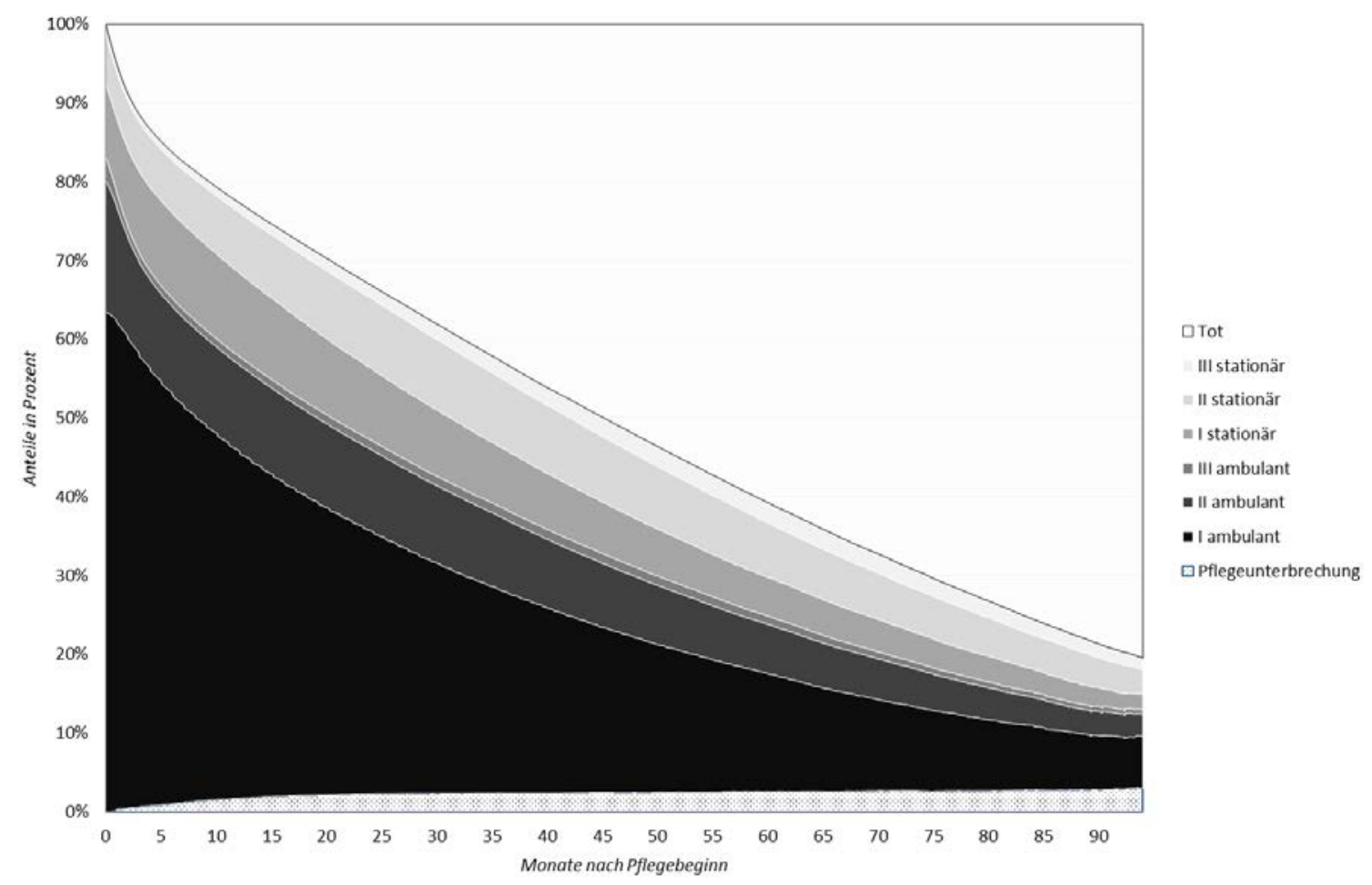

(a) Frauen $(\mathrm{N}=150.099)$

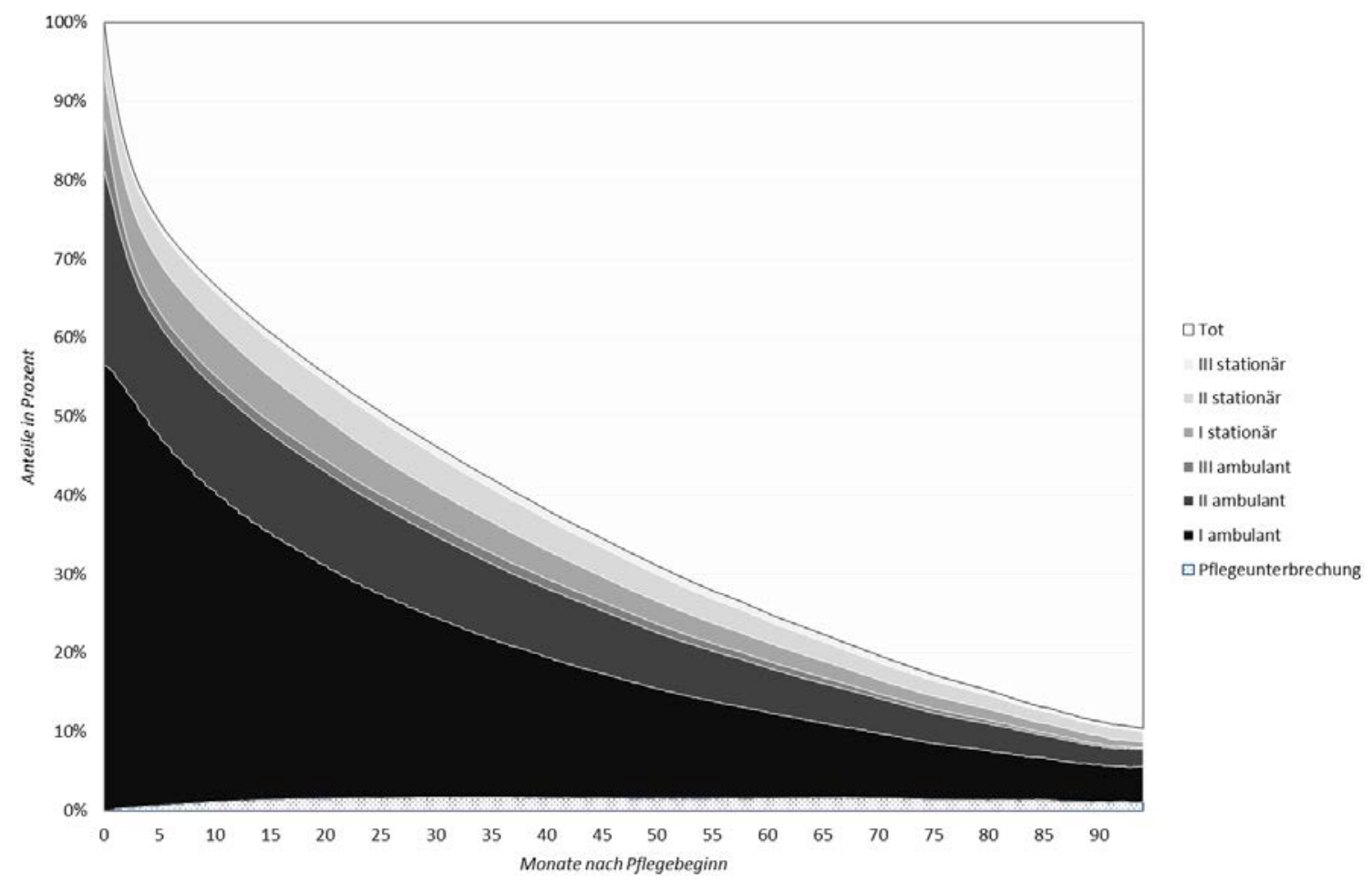

(b) Männer ( $N=71.892)$

Quelle: eigene Berechnungen 
Betrachtet man den Pflegeverlauf differenziert nach der Ersteinstufung in die Pflege (vgl. Abbildung 5, Abbildung 6 und Abbildung 7 im Anhang), so sind Pflegeunterbrechungen vor allem bei jenen Personen zu finden, die ihren Pflegeverlauf mit Pflegestufe I beginnen. Demnach befinden sich nach 94 Monaten bei den Frauen (Männern) ca. 4 (2) Prozent der Pflegefälle in einer Pflegeunterbrechung. Bei weiblichen (männlichen) Pflegefällen, die direkt mit Pflegestufe II eingestuft werden, ist dies nach dem gleichen Zeitraum nur bei ca. 1,5 (0,5) Prozent der Fälle zu beobachten, wohingegen in Pflegestufe III keine Reaktivierung stattfindet. In Einklang mit diesen Ergebnissen steht auch die Entwicklung der Sterblichkeit. So sinkt der Zeitpunkt, an dem 50 Prozent der weiblichen (männlichen) Pflegefälle verstorben sind, von 51 (36) Monaten auf 21 (8) Monate und schließlich $2(1,5)$ Monate ab, je nachdem ob die Pflege mit Pflegestufe I, II oder III begonnen hat.

Eine Betrachtung der Sterblichkeit aufgeschlüsselt nach der Erstjahres- und Folgejahressterblichkeit, dem Alter sowie der Ersteinstufung in die ambulante oder stationäre Pflege gibt weitere Aufschlüsse über die Entwicklung der Pflegeverläufe (vgl. Tabelle 2, Tabelle 3 und Tabelle 4). ${ }^{16}$ So zeigt sich hier wiederum über alle Altersgruppen hinweg, dass die Erstjahressterblichkeit der Männer für alle Möglichkeiten der Ersteinstufung in die Pflege über der Sterblichkeit der Frauen liegt, wobei die Sterblichkeit für beide Geschlechter mit der Pflegstufe ansteigt.

Tabelle 2: Sterblichkeit bei Ersteinstufung in Pflegestufe I

\begin{tabular}{|c|c|c|c|c|}
\hline \multirow[t]{2}{*}{ Alter bei Pflegebeginn } & \multicolumn{2}{|c|}{ Männer } & \multicolumn{2}{|c|}{ Frauen } \\
\hline & Ambulant (N) & Stationär (N) & Ambulant (N) & Stationär (N) \\
\hline $60-69$ & $(4576)$ & (516) & $(4328)$ & (464) \\
\hline 70-79 & $20,4 \quad(16930)$ & $20,7 \quad(1568)$ & $12,7 \quad(29783)$ & $14,6 \quad(3657)$ \\
\hline $80-89$ & $20,6 \quad(16495)$ & $23,3 \quad(1901)$ & $12,9 \quad(51948)$ & $15,5 \quad(8071)$ \\
\hline $90+$ & $(2630)$ & (378) & (9110) & $19,3 \quad(1763)$ \\
\hline
\end{tabular}

Pflegestufe 1: Geometrisches Mittel der Sterblichkeit in den Folgejahren (Jahre: 2-8)

\begin{tabular}{|l|rrrrrrrr|r|}
\hline \multirow{3}{*}{ Alter bei Pflegebeginn } & \multicolumn{4}{|c|}{ Männer } & \multicolumn{4}{c|}{ Frauen } \\
& \multicolumn{4}{|c|}{ Ambulant (N) } & Stationär (N) & Ambulant (N) & Stationär (N) \\
\cline { 2 - 9 } $\mathbf{7 0 - 7 9}$ & 14,0 & $(4576)$ & 12,9 & $(516)$ & 8,9 & $(4328)$ & 10,6 & $(464)$ \\
$\mathbf{8 0 - 8 9}$ & 19,7 & $(16930)$ & 21,8 & $(1568)$ & 12,8 & $(29783)$ & 14,0 & $(3657)$ \\
$\mathbf{9 0 +}$ & 27,5 & $(16495)$ & 26,3 & $(1901)$ & 19,7 & $(51948)$ & 22,1 & $(8071)$ \\
\hline
\end{tabular}

Quelle: eigene Berechnungen

\footnotetext{
${ }^{16}$ Die Auswertungen erfolgen angelehnt an das Vorgehen von Seger et al. (2011). Für jede der genannten Kombinationen von Alter und Pflegestufe wird dabei eine separate Überlebensfunktion geschätzt. Die Erstjahressterblichkeit und die Sterblichkeit in den Folgejahren werden dann anhand der resultierenden Überlebensfunktion bestimmt.
} 
Als ein neuer Aspekt kommt jedoch auch zum Vorschein, dass die Erstjahressterblichkeit im ambulanten Bereich in Pflegestufe II und III für beide Geschlechter über der stationären Erstjahressterblichkeit liegt. Mithin scheint also bei gleicher Pflegestufe ein schwerwiegenderer Krankheitsverlauf bei den ambulant Gepflegten vorzuliegen, was zum einen auf eine (SelbstJSelektion der Pflegepatienten oder auch eine bessere Betreuung in den Pflegeheimen zurückgeführt werden könnte. Die Sterblichkeit in den Folgejahren liegt für beide Geschlechter, alle Pflegestufen sowie alle Pflegearrangements unter der Erstjahressterblichkeit, wobei sich hier ein Alterstrend abzeichnet. So steigt die Folgejahressterblichkeit in allen beobachteten Szenarien mit dem Alter an. Bei der Erstjahressterblichkeit zeigt sich hingegen ein durchmischtes Bild. So steigt die Erstjahressterblichkeit beispielsweise bei Männern, die stationär in Pflegestufe II eingestuft werden, mit dem Alter an, wohingegen in Pflegestufe III ambulant kein Anstieg der Erstjahressterblichkeit bei den Männern zu beobachten ist.

Tabelle 3: Sterblichkeit bei Ersteinstufung in Pflegestufe II

\begin{tabular}{|c|c|c|c|c|}
\hline \multicolumn{5}{|c|}{ Pflegestufe 2: Erstjahressterblichkeit } \\
\hline \multirow[t]{2}{*}{ Alter bei Pflegebeginn } & \multicolumn{2}{|c|}{ Männer } & \multicolumn{2}{|c|}{ Frauen } \\
\hline & Ambulant $(\mathrm{N})$ & Stationär (N) & Ambulant $(\mathrm{N})$ & $\begin{array}{c}\text { Stationär } \\
(\mathrm{N})\end{array}$ \\
\hline $60-69$ & $56,5 \quad(2453)$ & (431) & $(1663)$ & (411) \\
\hline 70-79 & $56,9 \quad(7759)$ & $45,8 \quad(1437)$ & (8459) & $34,1 \quad(2865)$ \\
\hline $80-89$ & $56,3 \quad(6515)$ & $48,8 \quad(1383)$ & $43,1 \quad(12061)$ & $35,9 \quad(5107)$ \\
\hline $90+$ & $61,3 \quad(1061)$ & (283) & $(2850)$ & $40,4 \quad(1206)$ \\
\hline
\end{tabular}

\begin{tabular}{|c|c|c|c|c|}
\hline \multirow[t]{2}{*}{ Alter bei Pflegebeginn } & \multicolumn{2}{|c|}{ Männer } & \multicolumn{2}{|c|}{ Frauen } \\
\hline & Ambulant (N) & Stationär (N) & Ambulant (N) & $\begin{array}{c}\text { Stationär } \\
(\mathrm{N})\end{array}$ \\
\hline $60-69$ & $22,1 \quad(2453)$ & $20,5 \quad(431)$ & $(1663)$ & (411) \\
\hline $70-79$ & $29,7 \quad(7759)$ & $27,4 \quad(1437)$ & (8459) & $19,5 \quad(2865)$ \\
\hline $80-89$ & $35,9 \quad(6515)$ & $43,3 \quad(1383)$ & $28,8 \quad(12061)$ & $26,8 \quad(5107)$ \\
\hline $90+$ & z kl. N (1061) & z kl. N & (2850) & $35,0 \quad(1206)$ \\
\hline
\end{tabular}

Quelle: eigene Berechnungen 
Tabelle 4: Sterblichkeit bei Ersteinstufung in Pflegestufe III

\begin{tabular}{|c|c|c|c|c|c|c|c|c|}
\hline \multicolumn{9}{|c|}{ Pflegestufe 3: Erstjahressterblichkeit } \\
\hline \multirow[t]{2}{*}{ Alter bei Pflegebeginn } & \multicolumn{4}{|c|}{ Männer } & \multicolumn{4}{|c|}{ Frauen } \\
\hline & \multicolumn{2}{|c|}{ Ambulant $(\mathrm{N})$} & \multicolumn{2}{|c|}{ Stationär (N) } & \multicolumn{2}{|c|}{ Ambulant (N) } & \multicolumn{2}{|c|}{ Stationär (N) } \\
\hline $60-69$ & 83,9 & (937) & 62,5 & $(152)$ & 80,0 & $(510)$ & 50,9 & $(127)$ \\
\hline $70-79$ & 85,6 & (2175) & 65,2 & (444) & 79,1 & $(1715)$ & 50,5 & (657) \\
\hline $80-89$ & 84,3 & (1296) & 73,1 & (315) & 77,6 & (1816) & 55,1 & (954) \\
\hline $90+$ & 85,3 & (199) & 74,1 & (58) & 78,3 & $(383)$ & 64,5 & $(191)$ \\
\hline
\end{tabular}

\begin{tabular}{|c|c|c|c|c|}
\hline \multirow[t]{2}{*}{ Alter bei Pflegebeginn } & \multicolumn{2}{|c|}{ Männer } & \multicolumn{2}{|c|}{ Frauen } \\
\hline & Ambulant (N) & Stationär (N) & Ambulant (N) & Stationär (N) \\
\hline $60-69$ & (937) & $19,4 \quad(152)$ & $(510)$ & $11,1 \quad(127)$ \\
\hline 70-79 & $39,0 \quad(2175)$ & $33,6 \quad(444)$ & $27,5 \quad(1715)$ & $24,1 \quad(657)$ \\
\hline $80-89$ & z kl. N (1296) & z kl. N (315) & $35,7 \quad(1816)$ & $31,6 \quad(954)$ \\
\hline $90+$ & $\mathrm{zkl} . \mathrm{N}$ & $\mathrm{z} \mathrm{kl.} \mathrm{N}$ & $\mathrm{z} \mathrm{kl.} \mathrm{N}$ & z kl. N (191) \\
\hline
\end{tabular}

Quelle: eigene Berechnungen

\subsection{Pflegeverweildauern und die Kosten der Pflege}

Da zum Ende des Beobachtungszeitraums noch 19 (10) Prozent der weiblichen (männlichen) Pflegepatienten am Leben sind, muss zur Berechnung der durchschnittlichen Verweildauer in der Pflege eine Modellrechnung zum weiteren Pflegeverlauf nach dem Ende unseres Beobachtungszeitraums vorgenommen werden. Als Grundlage für die Modellierung werden hierzu die Übergangswahrscheinlichkeiten, die sich aus dem Datensatz ergeben, herangezogen. Die Übergangswahrscheinlichkeiten lassen sich dabei entweder täglich, monatlich oder jährlich für die acht Beobachtungsjahre berechnen, wobei für eine erste Übersicht zunächst die durchschnittlichen jährlichen Übergangswahrscheinlichkeiten in Tabelle 5 dargestellt sind. Für die Frauen zeigt sich hierbei, dass das Verbleiben in einer Pflegestufe am wahrscheinlichsten ist, wobei die Verweilwahrscheinlichkeit mit höherer Pflegestufe abnimmt. Auch bei den Männern trifft diese Aussage qualitativ zu, wobei in Pflegestufe III die Sterbewahrscheinlichkeit überwiegt. Der Wechsel in eine niedrigere Pflegestufe ist für beide Geschlechter hingegen vernachlässigbar, wie ein Blick auf das untere Dreieck der Übergangsmatrix zeigt. Einzig die Übergangswahrscheinlichkeit aus der ambulanten Pflegestufe I und II in eine Pflegeunterbrechung liegt im niedrigen einstelligen Prozentbereich. 
Tabelle 5: Jährliche Übergangswahrscheinlichkeiten in Prozent

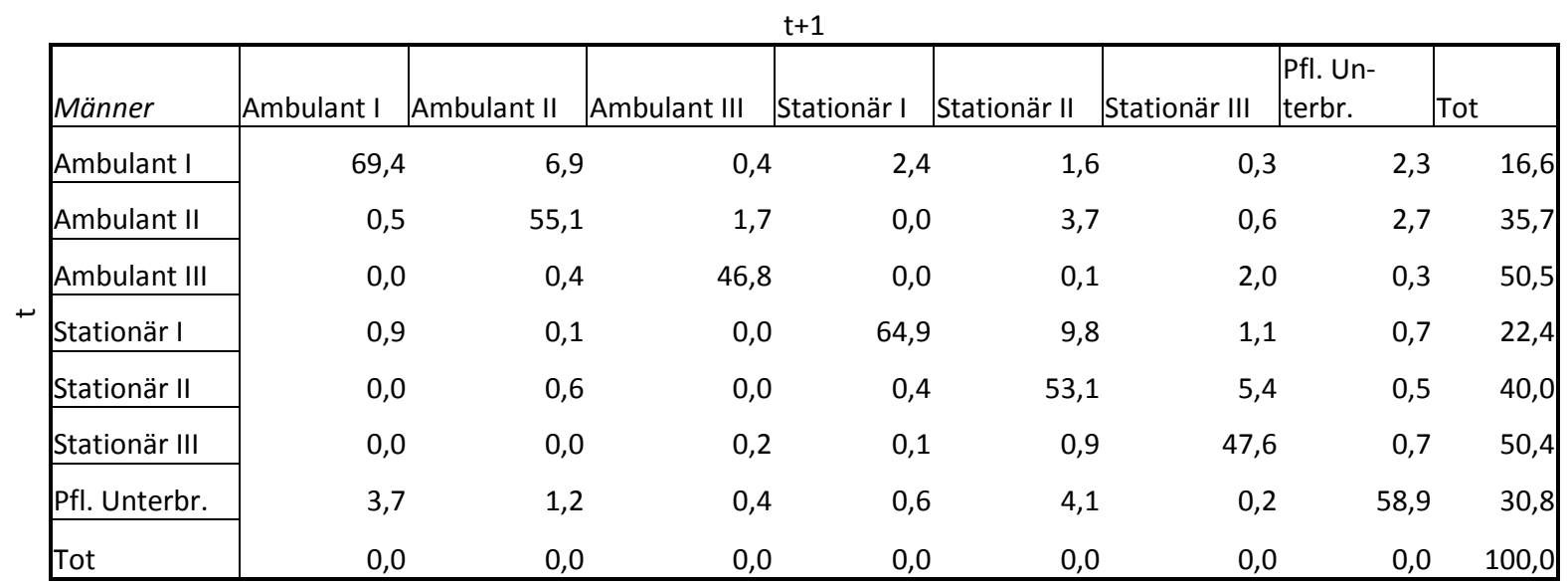

\begin{tabular}{|c|c|c|c|c|c|c|c|c|c|}
\hline Frauen & Ambulant I & Ambulant II & Ambulant III & Stationär I & Stationär II & Stationär III & $\begin{array}{l}\text { Pfl. Un- } \\
\text { terbr. }\end{array}$ & & Tot \\
\hline Ambulant I & 72,1 & 6,2 & 0,3 & 4,4 & 2,4 & 0,3 & & 3,2 & 11,1 \\
\hline Ambulant II & 0,6 & 57,9 & 2,2 & 0,1 & 6,1 & 0,8 & & 4,3 & 28,0 \\
\hline Ambulant III & 0,0 & 0,4 & 52,4 & 0,0 & 0,1 & 2,8 & & 0,2 & 44,2 \\
\hline Stationär I & 0,6 & 0,1 & 0,0 & 67,0 & 13,7 & 1,3 & & 0,5 & 16,8 \\
\hline Stationär II & 0,0 & 0,3 & 0,0 & 0,5 & 61,3 & 6,9 & & 0,5 & 30,5 \\
\hline Stationär III & 0,0 & 0,0 & 0,3 & 0,1 & 1,1 & 57,8 & & 0,7 & 40,0 \\
\hline Pfl. Unterbr. & 2,6 & 3,5 & 0,2 & 1,7 & 3,4 & 0,5 & & 64,8 & 23,2 \\
\hline Tot & 0,0 & 0,0 & 0,0 & 0,0 & 0,0 & 0,0 & & 0,0 & 100,0 \\
\hline
\end{tabular}

Quelle: eigene Berechnungen

Dem Vorgehen von Hackmann und Häcker (2012) folgend, werden für die Fortschreibung der Überlebensfunktionen aus Abbildung 1 die durchschnittlichen Übergangswahrscheinlichkeiten des achten Jahres in der Pflege verwendet. Im Weiteren wird dabei unterstellt, dass die Sterblichkeit aus den einzelnen Pflegestufen nicht mehr weiter ansteigt, sondern konstant auf dem Niveau des achten Jahres verharrt. Wie Abbildung 8 im Anhang zeigt, scheint diese Annahme gerechtfertigt, zeigt sich doch nach 24 Monaten in der Pflege kaum noch eine Veränderung in den monatlichen Mortalitätsraten. Die Fortschreibung der Überlebensfunktion erfolgt bis 300 Monate nach Pflegebeginn und sichert so ab, dass zum Ende der Fortschreibung weniger als 0,1 Prozent der zu Pflegenden in der Pflege verharren. Das Ergebnis dieser Fortschreibung ist schließlich in Abbildung 9 im Anhang dokumentiert.

Berechnet man die Flächen unter den fortgeschriebenen Überlebensfunktionen in Abbildung 9, erhält man die durchschnittliche Verweildauer in der Pflege. Tabelle 6 zeigt diese aufgetrennt nach Geschlecht und dem Anteil der jeweiligen Pflegestufen. Ein weiblicher (männlicher) Pflegefall über 60 Jahre verbleibt demnach durchschnittlich 51,4 $(36,6)$ Monate in 
Pflege, wobei mehr als 50 Prozent der Pflegezeit im Durchschnitt ambulant verbracht wird. Die Berechnung der durchschnittlichen Verweildauer hebt dabei zum einen hervor, dass die Verteilung der Pflegeverläufe extrem schief ist, zum anderen zeigt sich aber auch, dass nach 10 Jahren der Pflege noch ca. 10 (5) Prozent der weiblichen (männlichen) Pflegefälle in der Pflege verharren (vgl. Abbildung 9). Für einen gewissen Teil der Bevölkerung besteht somit das Risiko sehr lange in der Pflege zu verbleiben, was auch zu wesentlichen ökonomischen Belastungen der betroffenen Personen bzw. ihrer Angehörigen führen dürfte.

Tabelle 6: Durchschnittliche Verweildauer in der Pflege

\begin{tabular}{|c|c|c|}
\hline & Männer & Frauen \\
\hline Monate Total & 36,6 & 51,4 \\
\hline Anteil ambulant I & $48 \%$ & $38 \%$ \\
\hline Anteil ambulant II & $23 \%$ & $16 \%$ \\
\hline Anteil ambulant III & $3 \%$ & $3 \%$ \\
\hline Anteil stationär I & $10 \%$ & $15 \%$ \\
\hline Anteil stationär II & $12 \%$ & $20 \%$ \\
\hline Anteil stationär III & $4 \%$ & $8 \%$ \\
\hline
\end{tabular}

Quelle: eigene Berechnungen

Um ein Bild dieser ökonomischen Belastungen zu bekommen, wird im Weiteren mittels einer Monte-Carlo-Simulation ein künstlicher Datensatz erzeugt, in dem die Pflegepatienten durch die monatlichen Übergangsmatrizen wandern, die aus der vorhergehenden Analyse gewonnen wurden. Anschließend können dann durchschnittliche Pflegekosten berechnet werden. Als Kosten werden hierbei die reinen Pflegekosten zu Grunde gelegt, wie sie sich in Tabelle 7 darstellen, wobei in der ambulanten Pflege vereinfachend davon ausgegangen wird, dass nur Sachleistungen nachgefragt werden. So verursacht bspw. ein ambulanter Pflegefall in Pflegestufe I monatliche Kosten in Höhe von 694 Euro, wohingegen sich die Ausgaben im stationären Bereich mit Pflegestufe I auf 1855 Euro summieren. Nicht berücksichtigt werden bei den stationären Leistungen die Kosten für Unterkunft und Verpflegung, wohingegen die Investitionskosten einbezogen sind. Mithin wird in unseren Berechnungen davon ausgegangen, dass die 
Differenz zwischen stationären und ambulanten Unterkunfts- und Verpflegungskosten vernachlässigbar ist. Ebenfalls wird in den weiteren Berechnungen nicht berücksichtigt, dass auf einen Haushalt zusätzliche Ausgaben zukommen können, die sich bspw. dadurch ergeben, dass ein Partner weiterhin zu Hause wohnt, während der zu Pflegende in einem Heim untergerbacht wird.

Tabelle 7: Monatliche Pflegekosten im Jahr 2014 in Euro ${ }^{17}$

\begin{tabular}{|c|c|c|c|}
\hline & Gesamtpflegekosten & Sachleistung SPV & Selbstbehalt \\
\hline ambulant I & 694 & 450 & 244 \\
\hline ambulant II & 1.484 & 1.100 & 384 \\
\hline ambulant III & 2.003 & 1.550 & 453 \\
\hline stationär I & 1.855 & 1.023 & 832 \\
\hline stationär II & 2.317 & 1.279 & 1.038 \\
\hline stationär III & 2.806 & 1.550 & 1.256 \\
\hline
\end{tabular}

Quelle: Schneekloth und Schmidt (2011) ; Rothgang et al. (2013), BMG (2014)

Die Modellrechnung zu den Belastungen ist im Weiteren so aufgebaut, dass von einem Pflegebeginn im Jahr 2014 ausgegangen wird, wobei die Pflegeleistungen der SPV wie auch die Gesamtpflegekosten jährlich mit einer Wachstumsrate von 1,5 Prozent wachsen. Die anfallenden Beträge werden dann auf das Jahr 2014 mit einem Zinssatz von drei Prozent abgezinst.

Nach unseren Berechnungen betragen die Gesamtkosten der Pflege (Barwert) für die Frauen (Männer) im Mittelwert 68.871 (45.233) Euro, wobei der Median bei 50.674 (28.421) Euro liegt. Die rechtsschiefe Verteilung der Gesamtkosten ergibt sich dabei durch die zu Grunde liegende Lebenserwartung nach Pflegebeginn. Subtrahiert man von diesen Kosten die barwertigen Leistungen der SPV, so ergibt sich im Mittelwert ein Eigenanteil von 26.504 (16.225) Euro bei den Frauen (Männern), wobei der Median der selbst zu tragenden Kosten bei den Frauen (Männern) bei 17.395 (9.336) Euro liegt. Einschränkend sei darauf hingewiesen, dass die an-

\footnotetext{
${ }^{17}$ Die Gesamtpflegekosten wurden vom Basisjahr der Quelle mit einer Wachstumsrate von 1,5 Prozent bis ins Jahr 2014 fortgeschrieben.
} 
geführten Kostenberechnungen auf der Annahme fußen, dass die weiter steigende Lebenserwartung keinen Einfluss auf die Verweildauer in der Pflege hat. Inwieweit die vorliegenden Daten Aussagen hierüber erlauben, soll im folgenden Abschnitt geklärt werden.

\section{Die Auswirkungen einer längeren Lebenserwartung auf die Pflege}

Der Zusammenhang zwischen einer fortschreitenden Erhöhung der Lebenserwartung und den Auswirkungen auf den Pflegeverlauf wird zumeist anhand von drei Hypothesen diskutiert. ${ }^{18}$ So postuliert die Status-quo-Hypothese, dass die geschlechts- und altersspezifische Prävalenz der Pflege, bei einer zukünftig steigenden Lebenserwartung entsprechend der heute beobachtbaren Prävalenz verläuft. Die Medikalisierungsthese unterstellt hingegen, dass die Prävalenz der Pflege mit steigender Lebenserwartung überproportional ansteigt, da die hinzugewonnenen Lebensjahre zunehmend in einem multimorbiden Zustand verbracht werden. Im Gegensatz dazu bringt die Kompressionsthese zum Ausdruck, dass die Prävalenz der Pflege maßgeblich von der Nähe zum Todeszeitpunkt abhängt und zukünftig hinzugewonnene Lebensjahre somit zu keinem Anstieg in der Nachfrage nach Pflege führen. Die über diese sogenannte schwache Kompressionsthese hinausgehende starke Kompressionsthese stellt gar zur Debatte, dass mit zunehmendem Alter eine kürzere Verweildauer in der Pflege zu erwarten ist, was zu sinkenden pro-Kopf-Ausgaben in der Pflege führt.

Empirisch wurden diese Hypothesen bereits von verschiedenen Studien überprüft, wobei oftmals nicht nur die Pflege- sondern die gesamten Gesundheitsausgaben betrachtet werden. So kommen Werblow et al. (2007) auf der Basis von Schweizer Versicherungsdaten für die Jahre 1999 bis 2004 zu dem Ergebnis, dass das Alter insgesamt betrachtet keinen Einfluss auf die Gesundheitsausgaben hat, sondern vielmehr nur die Nähe zum Tod eine Rolle spielt. Betrachtet man jedoch die Pflegeausgaben für sich alleine, so finden die Autoren zumindest in diesem Teilbereich der Gesundheitsausgaben einen positiven Alterseffekt, der darauf hindeutet, dass ein hohes Alter die Wahrscheinlichkeit erhöht, Pflegeleistungen in Anspruch zu nehmen. Auch die Analysen von Weaver et al. (2009) haben zum Ergebnis, dass neben der Nähe zum Tod auch das Alter einen positiven Effekt darauf hat, ob eine Pflegeleistung in Anspruch genommen wird oder nicht. In Einklang mit diesen Ergebnissen präsentiert sich die Studie von Häcker und Hackmann (2012). Die Autoren kommen für bereits pflegebedürftige Personen auf der

\footnotetext{
${ }^{18}$ Eine Übersicht über die Hypothesen gibt beispielsweise Heigl (2002).
} 
Basis von Routinedaten der GKV zu dem Ergebnis, dass eine höhere Pflegestufe umso wahrscheinlicher ist, je näher der Abstand zum Tod und je höher das Alter ist, wobei sich für die Jahre 2000 bis 2009 insgesamt ein Zeittrend beobachten lässt, der einer höheren Pflegeeinstufung entgegenwirkt. Die Autoren führen an, dass dies auf eine strengere Einstufungspraxis des MDK zurückgehen könnte.

\subsection{Empirische Vorgehensweise}

Da unser Datensatz nur Personen berücksichtigt, die bereits in der Pflege sind, bietet sich im Weiteren eine Orientierung an dem empirischen Vorgehen von Häcker und Hackmann (2012) an, wobei die Daten hierzu auf Monatsebene betrachtet werden. ${ }^{19}$ Dem Vorgehen der genannten Autoren folgend, wurde zunächst eine Analyse mittels eines ordinalen logistischen Modells in Betracht gezogen. Wie sich für unsere Daten jedoch zeigt, wird dabei die zentrale Annahme der parallelen Wirkungsrichtung der unabhängigen Variablen verletzt. Im Folgenden wählen wir daher einen multinominalen logistischen Ansatz der folgenden Form: ${ }^{20}$

$$
\begin{aligned}
& g_{K}(x)=\ln \left[\frac{P(Y=K \mid x)}{P(Y=1 \mid x)}\right] \\
& =\propto_{K 0}+\propto_{K 1} \text { geschlecht }+\propto_{K 2} \text { stationär }+\propto_{K 3} \text { tot }+\propto_{K 4} \text { eintritt }+\propto_{K 5} \text { alter } \\
& +\propto_{K 6} \text { alter }^{2}+\propto_{K 7} \text { ttd }+\propto_{K 8} t t d^{2} ; \text { für } K=2,3
\end{aligned}
$$

Um den Gesundheitszustand zu approximieren, wird dabei die Pflegestufe I bis III als abhängige Variable herangezogen, wobei der Datensatz gepoolt analysiert wird. ${ }^{21}$ Als unabhängige Variablen wählen wir im Weiteren einen Dummy für das Geschlecht (geschlecht; Frauen=1), einen Dummy für die ambulante oder stationäre Pflege (stationär; stationär=1) sowie einen Dummy dafür, ob einer Person innerhalb des Beobachtungszeitraums verstirbt oder zensiert wird (tot; tot=1). Die Unterscheidung zwischen zensierten und verstorbenen Personen scheint uns dadurch gerechtfertigt, dass zum Ende unseres Beobachtungszeitraums ein relativ großer Teil unseres Personenkreises (ca. 37 Prozent) zensiert ist. Zudem nehmen wir in unsere Regression den Eintrittszeitpunkt in die Pflege auf (eintritt), um zu kontrollieren, ob ein zeitlicher Trend bei der Einstufung in die Pflegestufen vorliegt. Der Eintrittszeitpunkt in die Pflege wird

\footnotetext{
${ }^{19}$ Der Datensatz wird dazu vom long- in das wide-Format gebracht, wobei für jeden Monat in Pflege ein Spell erzeugt wird.

${ }^{20}$ Separate logistische Modelle wurden ebenfalls geschätzt. Die hieraus resultierenden Koeffizienten liegen den Koeffizienten aus dem multinominalen logistischen Modell sehr nahe. Ein Likelihood-Ratio-Test zeigt zudem, dass alle berücksichtigten, unabhängigen Variablen signifikant verschieden von 0 sind.

${ }^{21}$ Eine Panelanalyse, die beispielsweise auf fixen Effekten beruht, ist leider nicht möglich, da sich alter und $t t d$ für jeden Pflegepatienten zu einer zeitinvarianten Größe summieren.
} 
dabei als monatliche Variable kodiert, wobei der 1. Januar 2003 als Startzeitpunkt dient. Die für uns zentralen Variablen Alter (alter) und Zeit bis zum Tod (ttd) werden schließlich ebenfalls auf monatlicher Basis kodiert, wobei jeweils auch ein quadratischer Term berücksichtigt wird (alter2; $t t d 2) .{ }^{22}$ Sollte sich die Kompressionsthese bestätigen, so ist zu erwarten, dass eine höhere Pflegestufe umso unwahrscheinlicher wird, je größer der zeitliche Abstand zum Tod ist. Ein Alterseffekt, der darüber hinaus darauf hindeutet, dass eine höhere Pflegestufe mit zunehmendem Alter unwahrscheinlicher ist, würde zudem auf eine Kompression des Pflegeverlaufs bei einer steigenden Lebenserwartung hindeuten.

Alles in allem versuchen wir mit der beschriebenen Vorgehensweise herauszuarbeiten, inwieweit die verschiedenen Pflegestufen nach dem Eintritt in die Pflege durch die abhängigen Variablen erklärt werden können. Als Referenzkategorie dient uns hierzu die Pflegestufe I und II, wobei die Ergebnisse im Folgenden als relative Risk Ratios (Odds Ratios) dokumentiert werden.

\subsection{Ergebnisse}

Die Ergebnisse des multinominalen logistischen Modells sind in Tabelle 8 dargestellt. Für den Vergleich von Pflegestufe I und Pflegestufe II finden wir hierbei einen signifikanten Effekt für das Geschlecht, der zeigt, dass Männer gegenüber ihrer Referenzgruppe (Frauen) eine um 9 Prozent erhöhte Wahrscheinlichkeit aufweisen, in Pflegestufe II zu sein. Gerade anders herum verhält es sich für den Vergleich von Pflegestufe II und Pflegestufe III: Hier besteht für die Frauen eine um 8 Prozent erhöhte Wahrscheinlichkeit, in Pflegestufe III zu verweilen. Beim Vergleich von Pflegestufe I und Pflegestufe III zeigt sich hingegen kein signifikanter Unterschied zwischen den Geschlechtern.

Auch für den Dummy der stationären Pflege finden sich in unserem Modell signifikante Effekte. Diese deuten darauf hin, dass stationär Gepflegte gegenüber ambulant Gepflegten eine höhere Wahrscheinlichkeit aufweisen, in einer höheren Pflegstufe zu verharren. Im Detail bedeutet dies: Wird ein Pflegepatient stationär gepflegt, so ist die Wahrscheinlichkeit gegenüber seiner Referenzgruppe (ambulant Gepflegte), in Pflegestufe II oder III anstatt in Pflegestufe I zu sein, um das 2,9 bzw. 5,3 fache höher. Die Wahrscheinlichkeit sich als stationär Gepflegter

\footnotetext{
22 Jene Personen, die im Beobachtungszeitraum nicht versterben, weisen bei der Variable ttd keinen Eintrag auf. Dem Vorgehen von Werblow et al. (2011) folgend, wird für diese Personen daher angenommen, dass die Variable dem Maximum jener Personen entspricht, die versterben. Für den vorliegenden Datensatz beträgt dies 96 Monate. Vgl. hierzu auch Tabelle $10 \mathrm{im}$ Anhang.
} 
in Pflegestufe III anstatt in Pflegestufe II zu befinden, beträgt hingegen gegenüber seinem ambulanten Pendant nur das 1,8 fache.

Tabelle 8: Übergangswahrscheinlichkeiten in Pflegestufe II und III

Pflegestufe II vs. Pflegestufe I Pflegestufe III vs. Pflegestufe I Pflegestufe III vs. Pflegestufe II

\begin{tabular}{lccc} 
Variable & $\begin{array}{c}\text { Relative Risk Ratio } \\
\text { (Basis: Pflegestufe I) }\end{array}$ & $\begin{array}{c}\text { Relative Risk Ratio } \\
\text { (Basis: Pflegestufe I) }\end{array}$ & $\begin{array}{c}\text { Relative Risk Ratio } \\
\text { (Basis: Pflegestufe II) }\end{array}$ \\
\hline geschlecht & $0,9141087^{* * *}$ & 0,9919547 & $1,085161^{* * *}$ \\
eintritt & $(0,00189)$ & $(0,00409)$ & $(0,00453)$ \\
stationär & $0,9993734^{* * *}$ & $0,9922097^{* * *}$ & $0,9928317^{* * *}$ \\
& $(0,00005)$ & $(0,00010)$ & $(0,00010)$ \\
tot & $2,908855^{* * *}$ & $5,372047^{* * *}$ & $1,846791^{* * *}$ \\
& $(0,00571)$ & $(0,01972)$ & $(0,00681)$ \\
alter & 1,003801 & $0,7555653^{* * *}$ & $0,7527042^{* * *}$ \\
& $(0,00271)$ & $(0,00407)$ & $(0,00413)$ \\
alter2 & $0,9888557^{* * *}$ & $0,9857957^{* * *}$ & $0,9969055^{* * *}$ \\
& $(0,00020)$ & $(0,00037)$ & $(0,00038)$ \\
ttd & $1,000005^{* * *}$ & $1,000006^{* * *}$ & $1 *$ \\
& $(0,00000)$ & $(0,00000)$ & $(0,00000)$ \\
ttd2 & $0,9751872^{* * *}$ & $0,9477872^{* * *}$ & $0,9719029^{* * *}$ \\
& $(0,00013)$ & $(0,00024)$ & $(0,00025)$ \\
\hline$N=6771465)$ & $1,000236^{* * *}$ & $1,00014^{* * *}$
\end{tabular}

$\mathrm{N}=6771465$

Pseudo $R^{2}=0,0881$

*** signifikant auf $99 \%$ Niveau; Standardfehler in Klammern

Quelle: eigene Berechnungen

Wie aus der Literaturübersicht zu erwarten war, ist auch der Abstand zum Tod signifikant. Demnach sinkt die Wahrscheinlichkeit, in einer höheren Pflegestufe zu sein, mit zunehmender zeitlicher Entfernung vom Tod ab, sodass auch wir die Kompressionsthese bestätigen können. Ein genaues Bild, wie sich die Wahrscheinlichkeit, in Pflegestufe II oder III zu sein, im Verhältnis zu der Wahrscheinlichkeit in Pflegestufe I (II) zu verweilen (Chance (Odds)), ${ }^{23}$ entwickelt, bietet dabei Abbildung 2. ${ }^{24}$ So sinkt diese Chance mit zunehmendem Abstand vom Tod ab, wobei beispielsweise die Wahrscheinlichkeit sich in Pflegstufe II zu befinden, kurz vor dem Tod gleich hoch ist, wie die Wahrscheinlichkeit in Pflegstufe I zu verharren. Hingegen beträgt die geschätzte Wahrscheinlichkeit 80 Monate vor dem Tod in Pflegestufe II zu verweilen, nur ungefähr 25 Prozent der Wahrscheinlichkeit in Pflegestufe I zu sein. Die Chance in Pflegestufe IIII

\footnotetext{
${ }^{23}$ Chance $_{\text {Plfegestufe III vs. Pflegestufe II }}=\frac{\text { Wahrscheinlichkeit }_{\text {Pflegestufe III }}}{\text { Wahrscheinlichkeit }_{\text {Pflegestufe II }}}$

${ }^{24}$ Die anderen unabhängigen Variablen wurden dabei auf ihren Mittelwert fixiert. Vgl. hierzu Tabelle 10 im Anhang.
} 
anstatt in Pflegestufe I oder II zu sein, liegt für unsere Berechnungen hingegen durchweg unter dem Wert von eins.

\section{Abbildung 2: Chance des Wechsels zwischen den Pflegestufen unter Berücksichtigung des Zeit- raums bis zum Tod}

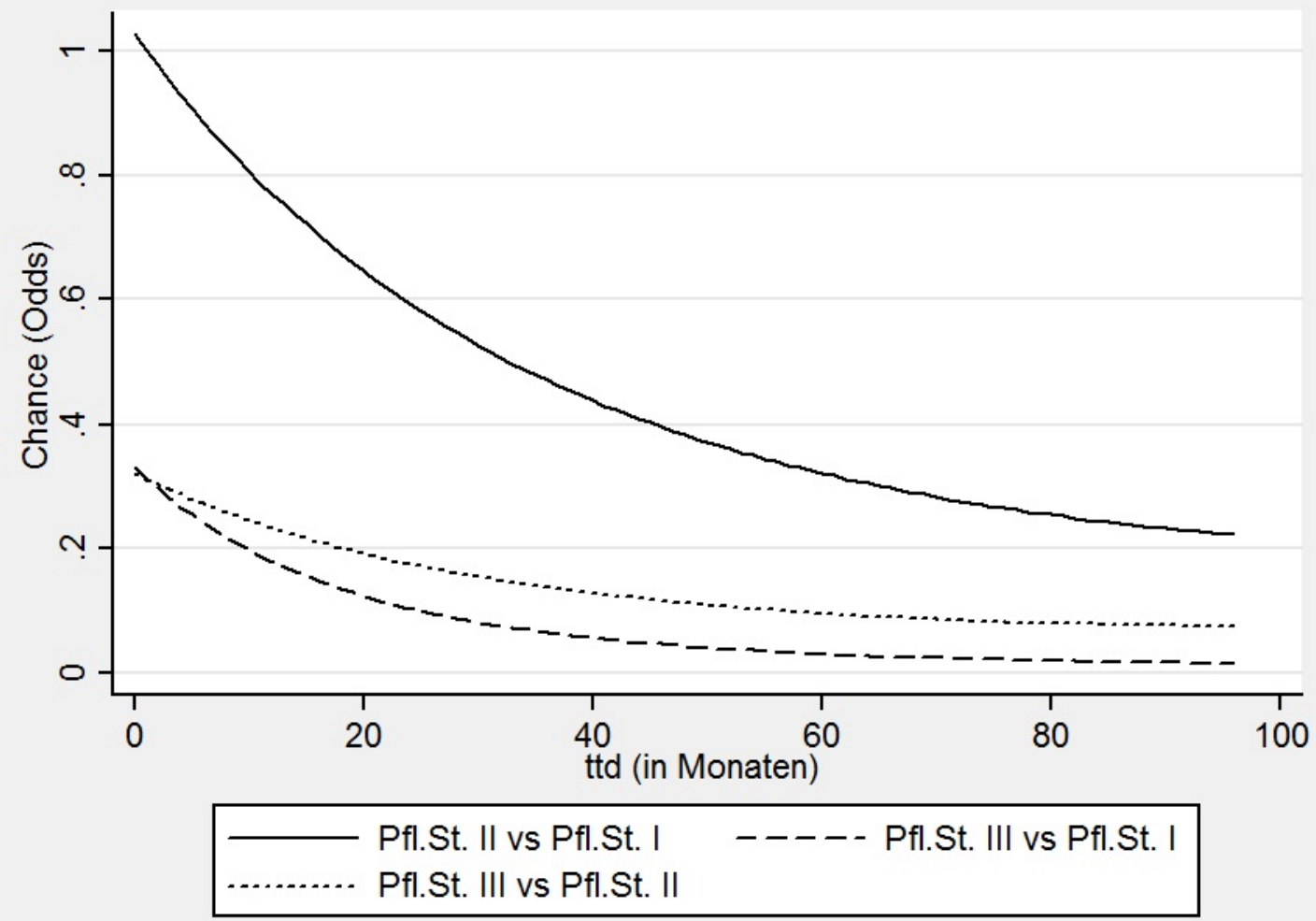

Quelle: eigene Berechnungen

Auch für das Alter finden wir einen signifikanten Effekt. Dieser deutet entgegen unserer Erwartungen jedoch darauf hin, dass die Wahrscheinlichkeit in einer höheren Pflegestufe zu sein, mit zunehmendem Alter abnimmt. Mithin legen unsere Daten also zumindest für den Pflegeverlauf nahe, dass der Anstieg der Lebenserwartung mit einer Verbesserung des Pflegeverlaufs einhergeht, wobei ungeklärt bleibt, wie die Veränderung der Lebenserwartung auf die Inzidenz der Pflege wirkt.

Abbildung 3 zeigt für die Variable alter, wie sich die Wahrscheinlichkeit, in Pflegestufe II oder III zu verharren, im Verhältnis zu der Wahrscheinlichkeit in Pflegestufe I (II) zu sein, entwickelt. Zunächst lässt sich festhalten, dass für alle betrachteten Kombinationen die Wahrscheinlichkeit der kleineren Pflegestufe überwiegt, wobei die Wahrscheinlichkeit in Pflegestufe III zu sein, gegenüber jener sich in Pflegestufe I (II) aufzuhalten monoton mit dem Alter abnimmt. Die Chance anstatt in Pflegestufe I in Pflegestufe II zu sein, hat hingegen einen U-förmigen Verlauf, wobei der Tiefpunkt ungefähr bei einem Alter von 87 Jahren liegt. 

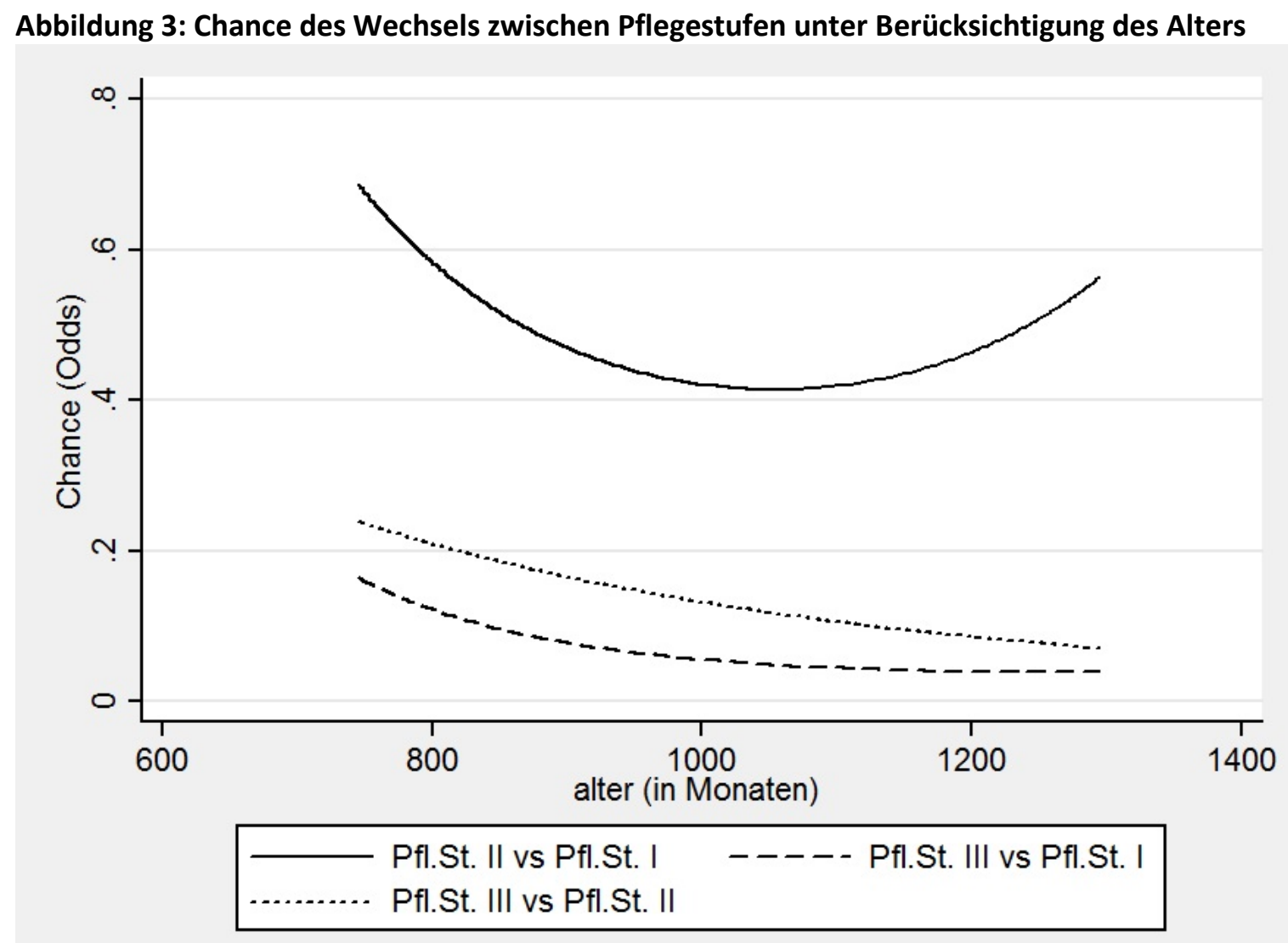

Quelle: eigene Berechnungen

Zuletzt finden auch wir einen signifikanten zeitlichen Trend in unseren Daten. Dieser deutet darauf hin, dass eine höhere Pflegestufe über die Zeit unwahrscheinlicher geworden ist. Ob dies, wie von Häcker und Hackmann (2012) postuliert, auf eine strengere Einstufungspraxis des MDK zurückgeht, oder ob hierbei andere Größen einen Einfluss haben, kann nicht abschließend beantwortet werden.

\section{Fazit}

Vorliegende Studie befasst sich auf der Basis von Routinedaten mit Pflegeverläufen in Deutschland. Wie die vorliegenden Analysen zeigen, liegt dabei eine rechtsschiefe Verteilung für beide Geschlechter vor. Demnach sind 50 Prozent der über 60-jährigen weiblichen (männlichen) Pflegefälle nach 45 (26) Monaten verstorben, wohingegen die mittlere Verweildauer in der Pflege bei 51,4 (36,6) Monaten liegt. Die Überlebensfunktionen zeigen darüber hinaus, dass die Mortalität im ersten Jahr der Pflege am höchsten ist. So sterben nach unseren Berechnungen 23 (35) Prozent der weiblichen (männlichen) Pflegefälle bereits in diesem Zeitraum, wobei hierfür vor allem jene Pflegefälle verantwortlich sind, die in Pflegestufe II oder III ersteingestuft werden. Betrachtet man den altersspezifischen Pflegeverlauf, so zeigt sich, dass 
vor allem die Sterblichkeit nach dem ersten Jahr der Pflege mit dem Alter zunimmt, wohingegen bei der Erstjahressterblichkeit ein heterogenes Bild vorliegt.

Die barwertigen Kosten, die aus diesem Pflegeverlauf resultieren, lassen sich mittels einer Monte-Carlo-Simulation quantifizieren. Generell folgt die Kostenverteilung dabei der geschätzten Überlebensfunktion, wobei vor allem jene Personen, die sehr lange in der Pflege verweilen, hohe Kosten verursachen. So liegen die Gesamtkosten der Pflege im Barwert für das oberste Dezil der Frauen (Männer) bei mehr als 161.000 (113.000) Euro. Zieht man hiervon die derzeitigen Leistungen der SPV ab, so verbleiben für diese Pflegefälle Kosten in Höhe von mehr als 66.000 (41.000) Euro im Barwert, die privat oder über eine private Pflegeversicherung zu schultern sind.

Die Auswirkungen des demografischen Wandels auf die Pflegeversicherung sind nach unseren Ergebnissen differenziert zu betrachten. So legen die vorliegenden Untersuchungen nahe, dass mit einer weiter steigenden Lebenserwartung tendenziell ein milderer Pflegeverlauf einhergeht. Demnach ist eine höhere Pflegestufe nicht nur mit zunehmendem Abstand vom Tod sondern darüber hinaus auch mit zunehmenden Alter unwahrscheinlicher, was auf ein Zutreffen der starken Kompressionsthese hindeutet. Die Frage, inwieweit diese Kompression auch für die Inzidenz der Pflege gilt, bleibt für unsere Untersuchungen jedoch unbeantwortet.

Insgesamt kann aus unseren Ergebnissen geschlussfolgert werden, dass eine mögliche nachhaltige Reform der sozialen Pflegeversicherung in die Richtung einer Hochrisikoversicherung gehen könnte. Das im Alter der Pflegefall eintritt, ist - und bleibt trotz der auch von uns gezeigten Kompression der Pflegeverläufe - sehr wahrscheinlich. Somit bietet sich hier eine Absicherung über eine private (aber evtl. staatlich verpflichtende) kapitalgedeckte Vorsorge an, die auch bei der Pflegeversicherung selbst angesiedelt sein könnte. Die soziale Pflegeversicherung könnte dann in ihrer bisherigen umlagefinanzierten Form als Hochrisikoversicherung für einerseits sehr harte Pflegefälle (Pflegestufe 3) und für anderseits sehr lange Pflegekarrieren aufkommen. Immerhin jede zehnte Frau und jeder zwanzigste Mann verweilen, wenn einmal in Pflege, dort für zehn Jahre. Hier erscheint eine soziale Absicherung sinnvoll und treffsicher. 


\section{Literaturverzeichnis}

Blinkert, B. und T. Klie (2001), Zukünftige Entwicklung des Verhältnisses von professioneller und häuslicher Pflege bei differierenden Arrangements und privaten Ressourcen bis zum Jahr 2050, Expertise im Auftrag der Enquête-Kommission Demografischer Wandel des Deutschen Bundestags, Wiesbaden.

BMG- Bundesministerium für Gesundheit (2011), Ratgeber zur Pflege. Alles, was Sie zur Pflege wissen müssen, Bundesministerium für Gesundheit, Berlin.

BMG- Bundesministerium für Gesundheit (2014), Pflegeversicherung im Überblick, Bundesministerium für Gesundheit, Berlin.

Borchert, L. und H. Rothgang (2006), Pflegeverläufe älterer Menschen. Eine deskriptive Längsschnittstudie über die Jahre 1998 bis 2004. In: Müller, R. und B. Braun (Hg.), Vom Quer zum Längsschnitt - Möglichkeiten der Analysen mit GKV-Daten, Sankt Augustin, 76-91.

Cleves, M.A. (2008), An introduction to survival analysis using Stata, Stata Press, Texas.

Heigl, A. (2002), Aktive Lebenserwartung: Konzeptionen und neuer Modellansatz, Zeitschrift für Gerontologie und Geriatrie, 35 (6), 519-527.

Häcker, J. und T. Hackmann (2012), Los(t) in long term care: Empirical Evidence from German Data 2000-2009, Health Economics, 21, 1427-1443.

MDK (2002), Pflegebericht des Medizinischen Dienstes Berichtszeitraum 2001/2002, Medizinischer Dienst der Spitzenverbände der Krankenkassen e.V., Essen.

MDK (2003), Pflegebericht des Medizinischen Dienstes 2003, Medizinischer Dienst der Spitzenverbände der Krankenkassen e.V., Essen.

Prüß, U., Küpper-Nybelen, J., Ihle, P. und I. Schubert (2006) , Verläufe von Pflegebedürftigkeit in Hessen in den Jahren 1999 bis 2002. Ergebnisse einer Längsschnittstudie, Gesundheitswesen, 68 (2), 123-127.

Rothgang, H., Iwansky, S., Müller, R., Sauer, S. und R. Unger (2011), Barmer GEK Pflegereport 2011. Zusätzliche Betreuungsleistungen für Personen mit erheblich eingeschränkter Alltagskompetenz, BARMER GEK, Schwäbisch Gmünd.

Rothgang H., Müller, R. und R. Unger (2013), BARMER GEK Pflegereport 2013 - Schwerpunktthema: Reha bei Pflege, BARMER GEK, Schwäbisch Gmünd.

Saal, T. (2012), Pflegeverläufe und ihre Determinanten - Eine empirische Analyse anhand von Routinedaten, unveröffentlichte Diplomarbeit, Freiburg.

Sala, C. und JP Raftery (2001), Econometric Issues in testing the age neutrality of health care expenditure, Health Economics, 10, 669-671.

Seger, W., Sittaro, N.-A., Lohse, R., Rabba, J. und J. Post (2008), Hannover Morbiditäts- und Mortalitäts-Pflegestudie(HMMPS): Langzeitverläufe, Pflegestufenübergänge und Reaktivierungen in der gesetzlichen Pflegeversicherung, Blätter der Deutschen Gesellschaft für Versicherungs- und Finanzmathematik e.V., 29, 29-43.

Seger, W., Sittaro, N.-A., Lohse, R. und J. Rabba (2011), Vergleich der Sterblichkeit ambulanter und stationärer Pflegepatienten im Langzeitverlauf. Hannover Morbiditäts- und MortalitätsPflegestudie, Deutsche Medizinische Wochenschrift, 136, 1465-1471. 
Schneekloth, U und M. Schmidt (2011), Abschlussbericht zur Studie "Wirkungen des PflegeWeiterentwicklungsgesetzes", Bundesministerium für Gesundheit, Berlin.

Schupp, J. und H. Künemund (2004), Private Versorgung und Betreuung von Pflegebedürftigen in Deutschland, DIW Wochenbericht, 20/2004, 289-294.

Weaver, F., Stearns, S. und EC. Norton (2009), Proximity to death and participation in the long term care market, Health Economics, 18, 867-883.

Werblow, A., Felder, S. und P. Zweifel (2007), Population ageing and health care expenditure: a school of red herrings, Health Economics, 16, 1109-1126. 


\section{Anhang}

Abbildung 4: Alter bei Pflegebeginn

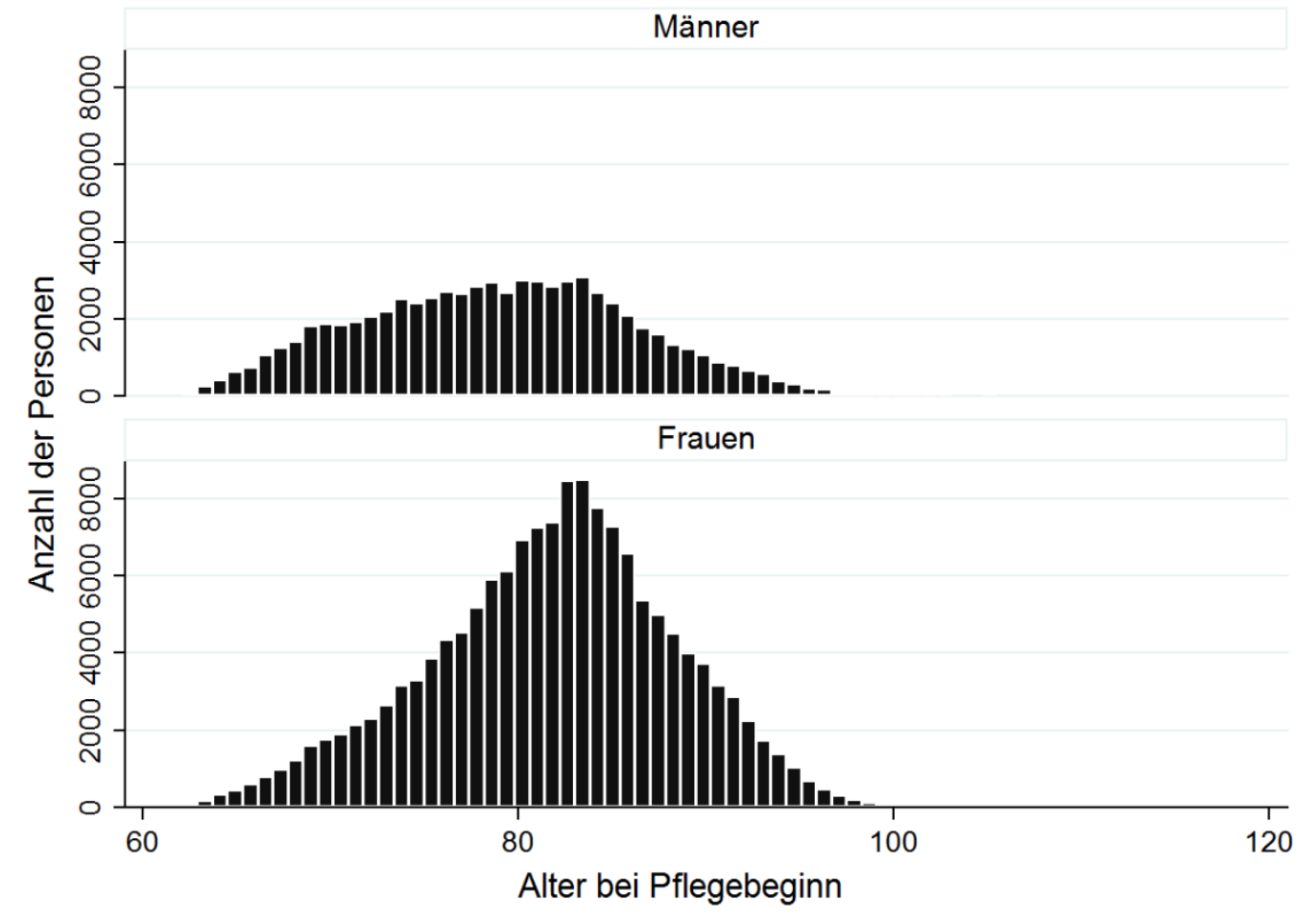

Quelle: eigene Berechnungen

Tabelle 9: Ersteinstufung in die Pflege im Jahr 2003

\begin{tabular}{|l|c|c|}
\hline \multicolumn{1}{|c|}{ Angaben in Prozent } & MDK & AOK-Plus \\
\hline Pflegestufe I & 65,9 & 66,9 \\
Pflegestufe II & 27,8 & 26,9 \\
\cline { 1 - 1 } Pflegestufe III & 6,3 & 6,2 \\
\hline
\end{tabular}

Quelle: MDK (2003), eigene Berechnungen 


\section{Abbildung 5: Pflegeverläufe nach Geschlecht und Ersteinstufung in Pflegestufe I}

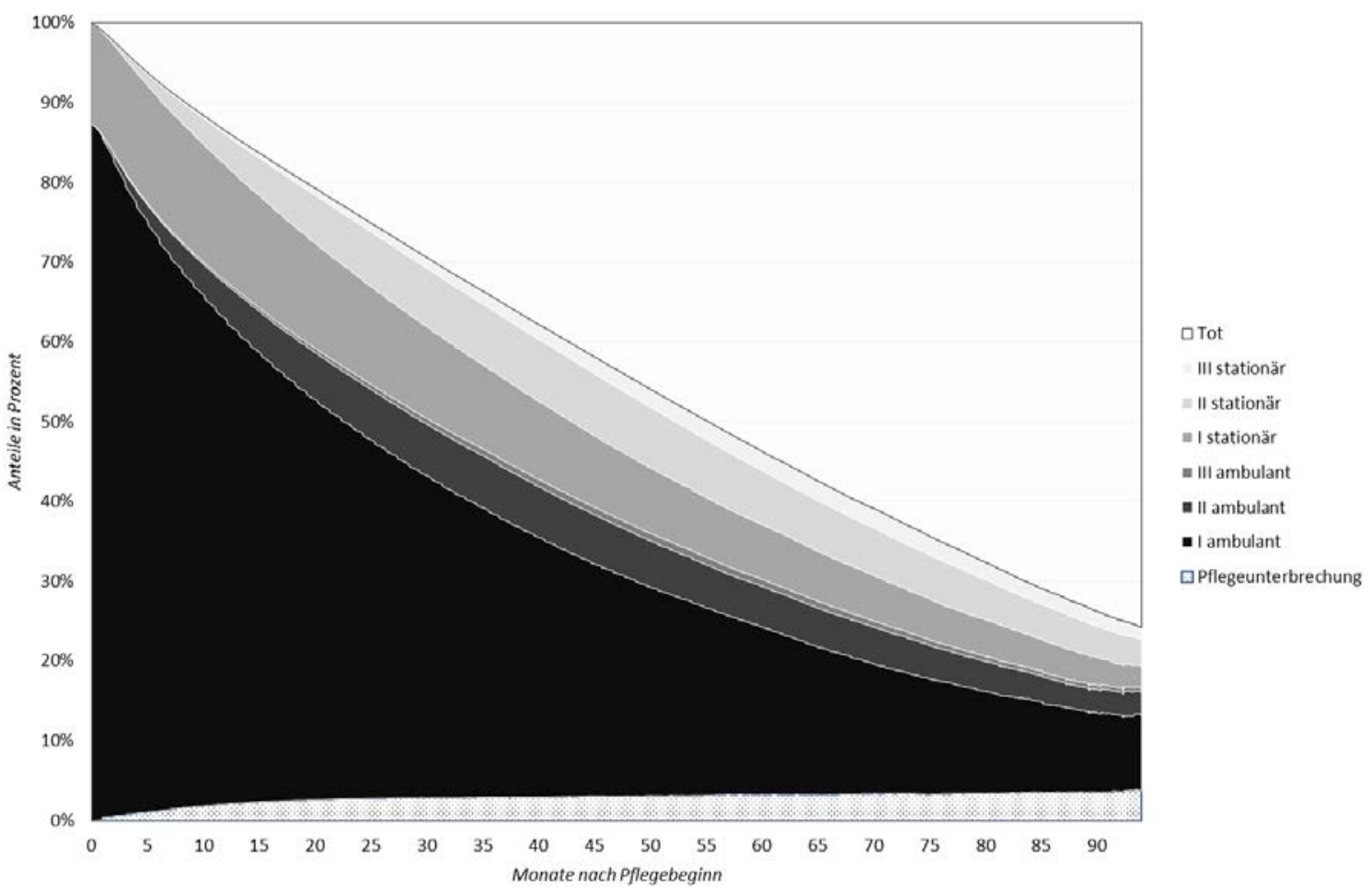

(a) Frauen $(\mathrm{N}=109.124)$

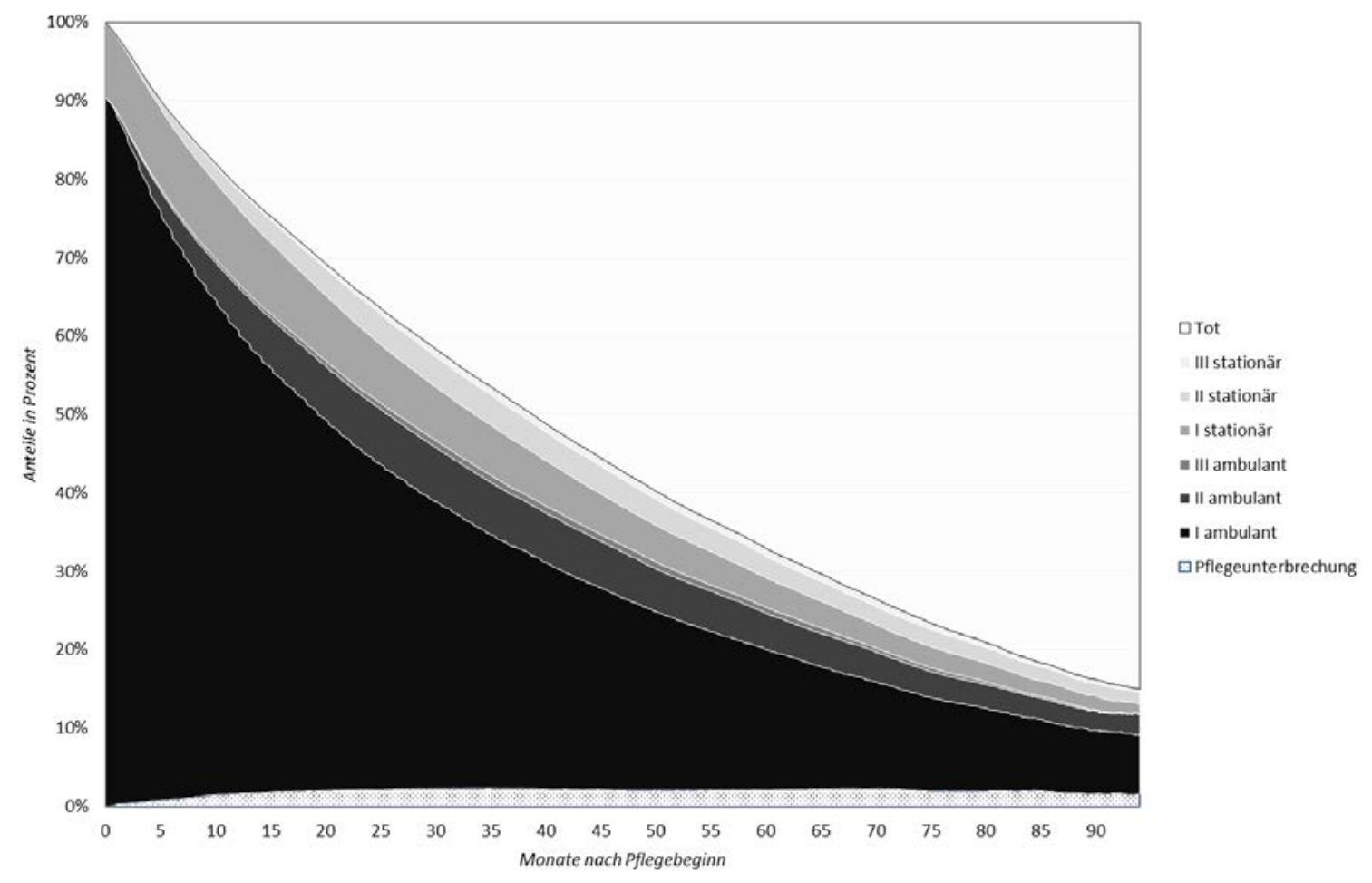

(b) Männer ( $N=44.994)$

Quelle: eigene Berechnungen 
Abbildung 6: Pflegeverläufe nach Geschlecht und Ersteinstufung in Pflegestufe II

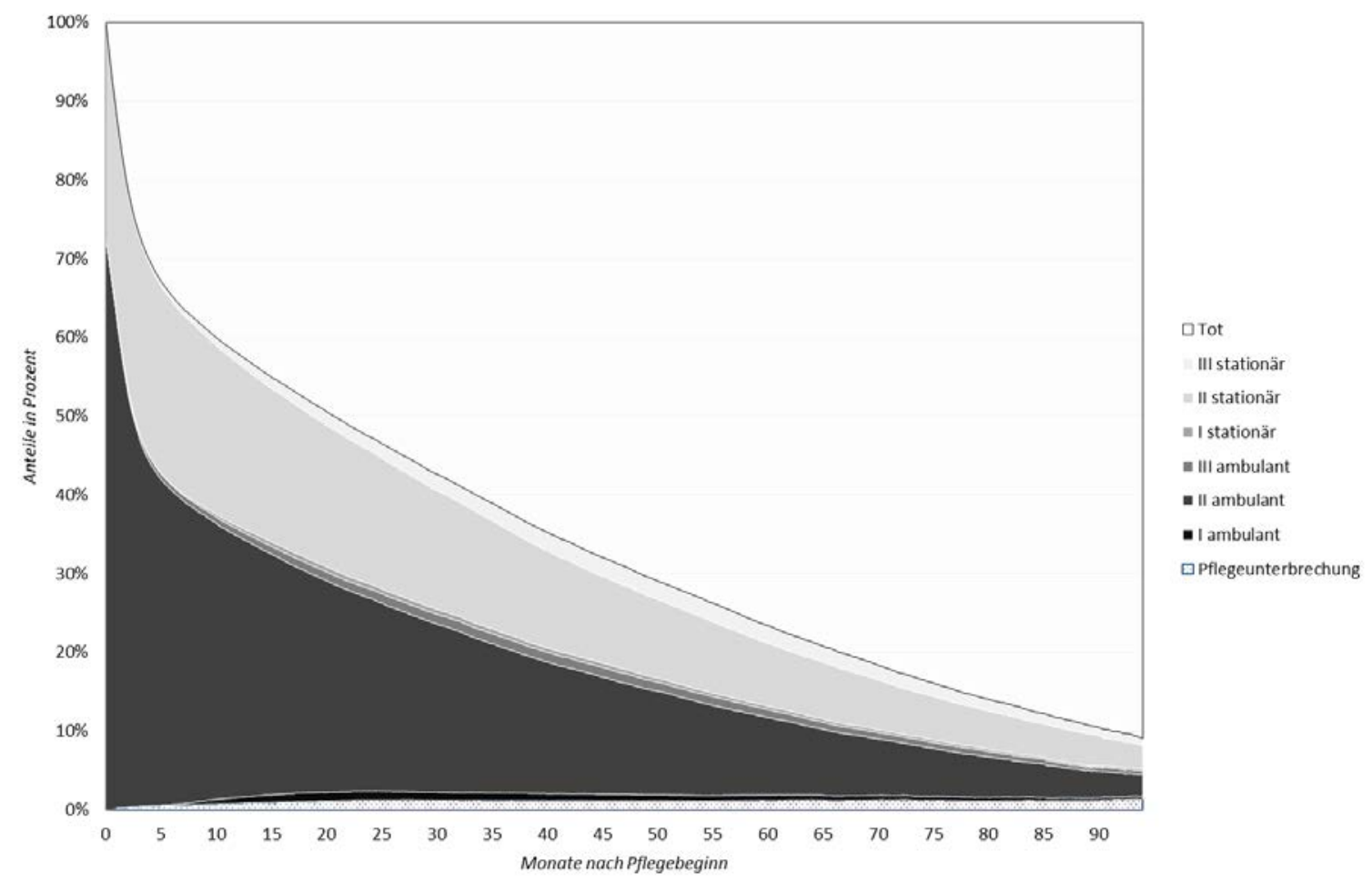

(a) Frauen ( $\mathrm{N}=34.622)$

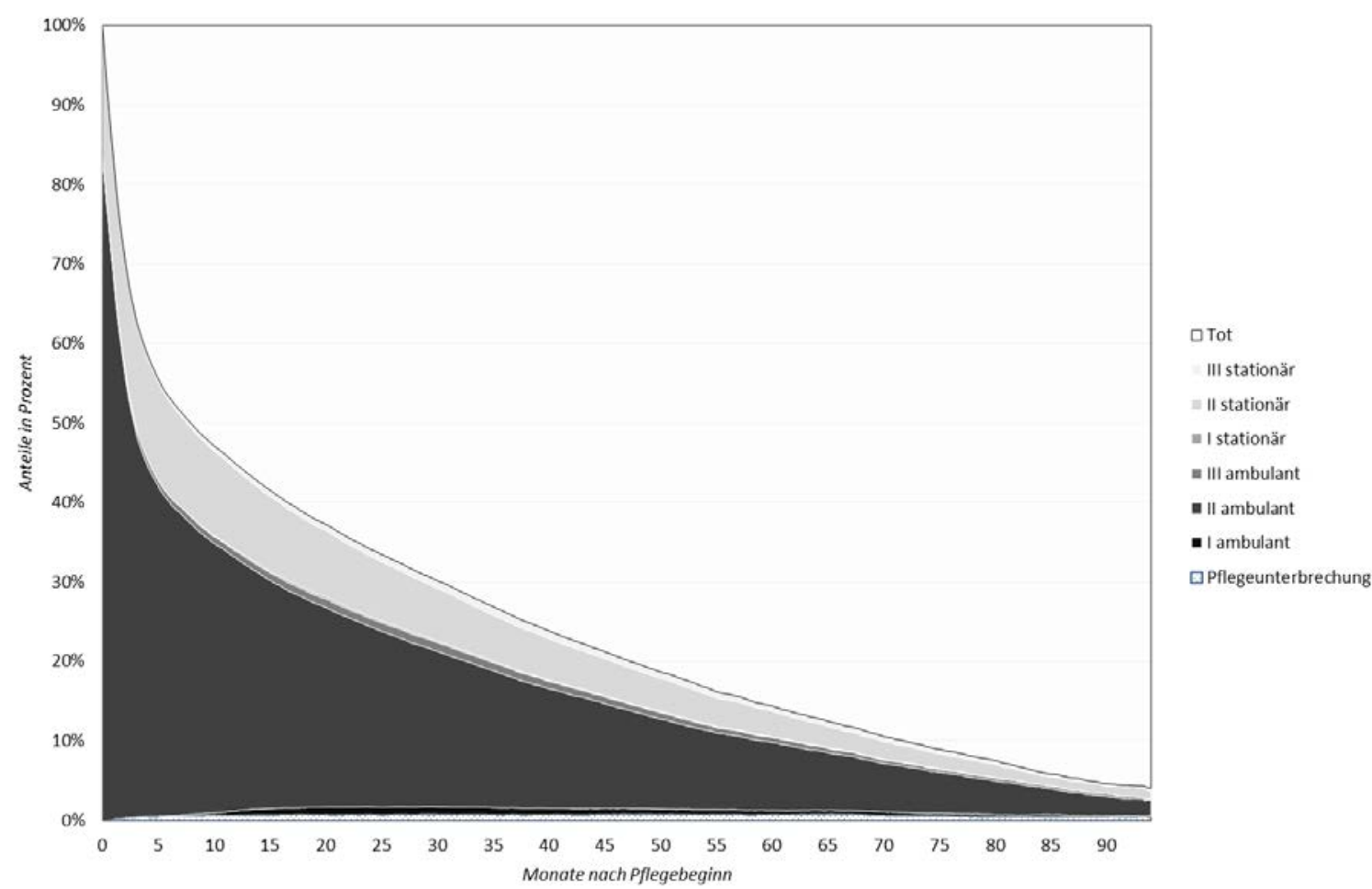

(b) Männer ( $N=21.322)$

Quelle: eigene Berechnungen 
Abbildung 7: Pflegeverläufe nach Geschlecht und Ersteinstufung in Pflegestufe III

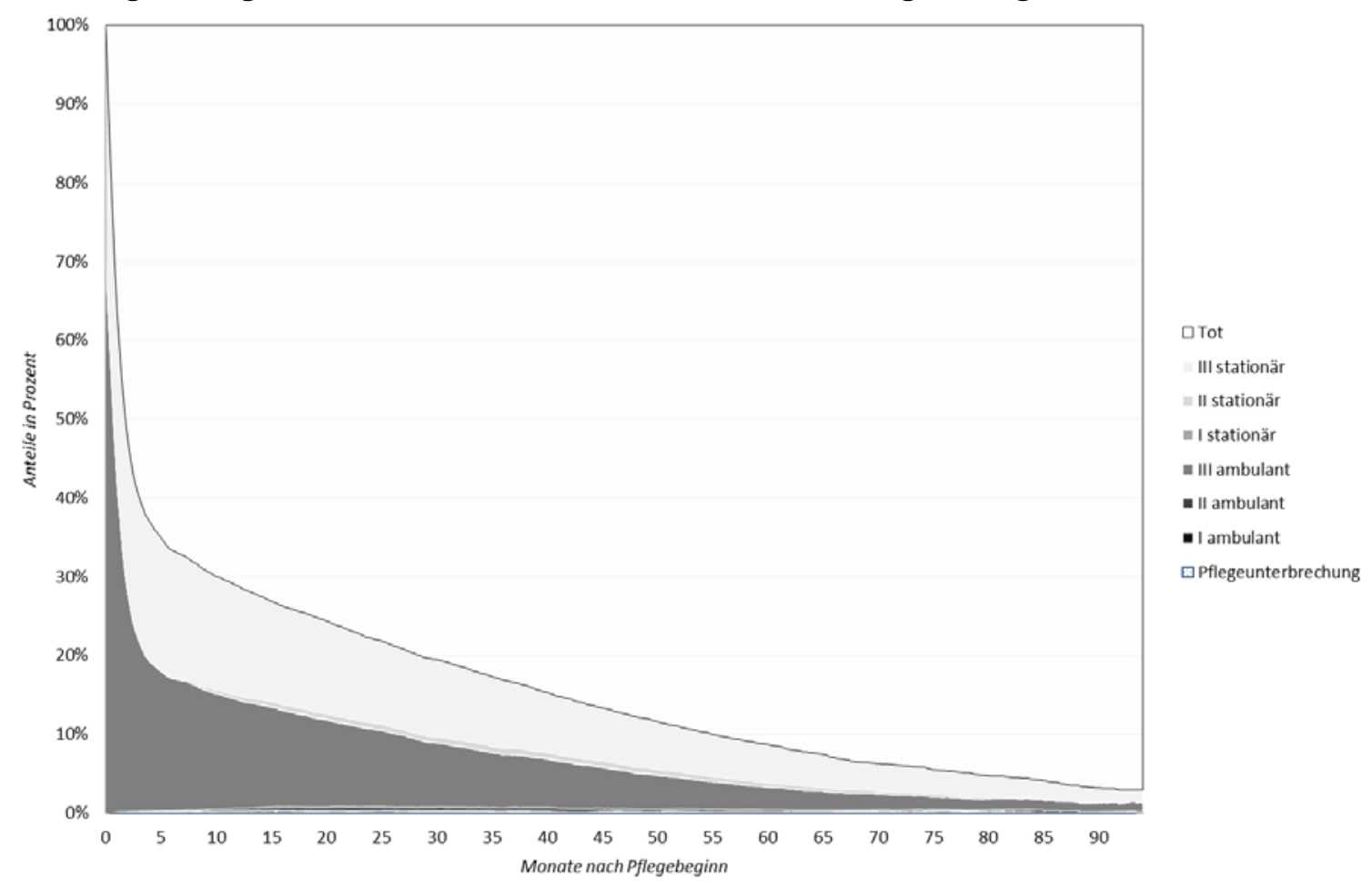

(a) Frauen $(\mathrm{N}=6.353)$

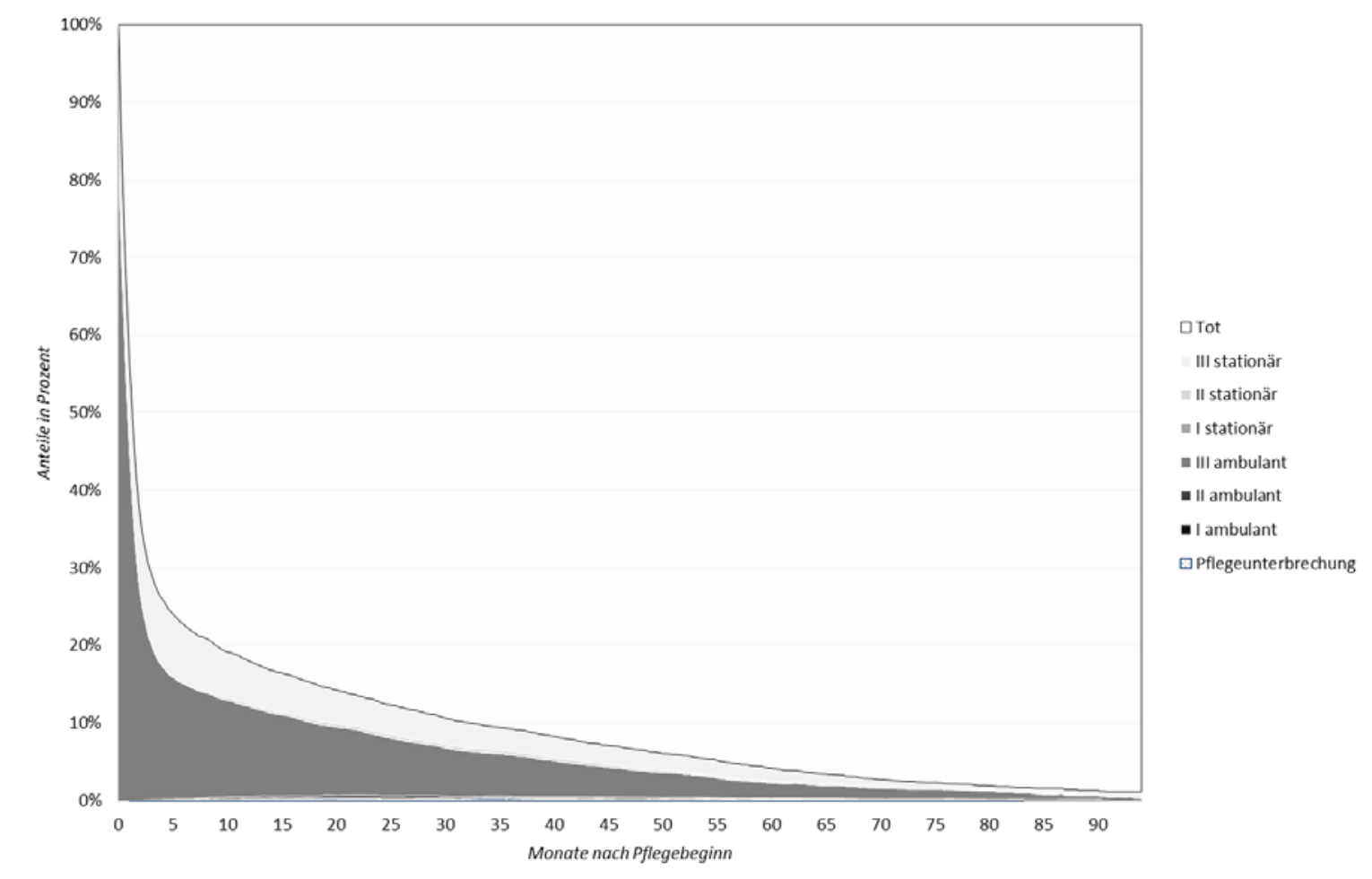

(b) Männer ( $N=5.576)$

Quelle: eigene Berechnungen 
Abbildung 8: Monatliche Mortalitätsraten aus den einzelnen Pflegestufen (gleitende 6-MonatsDurchschnitte)

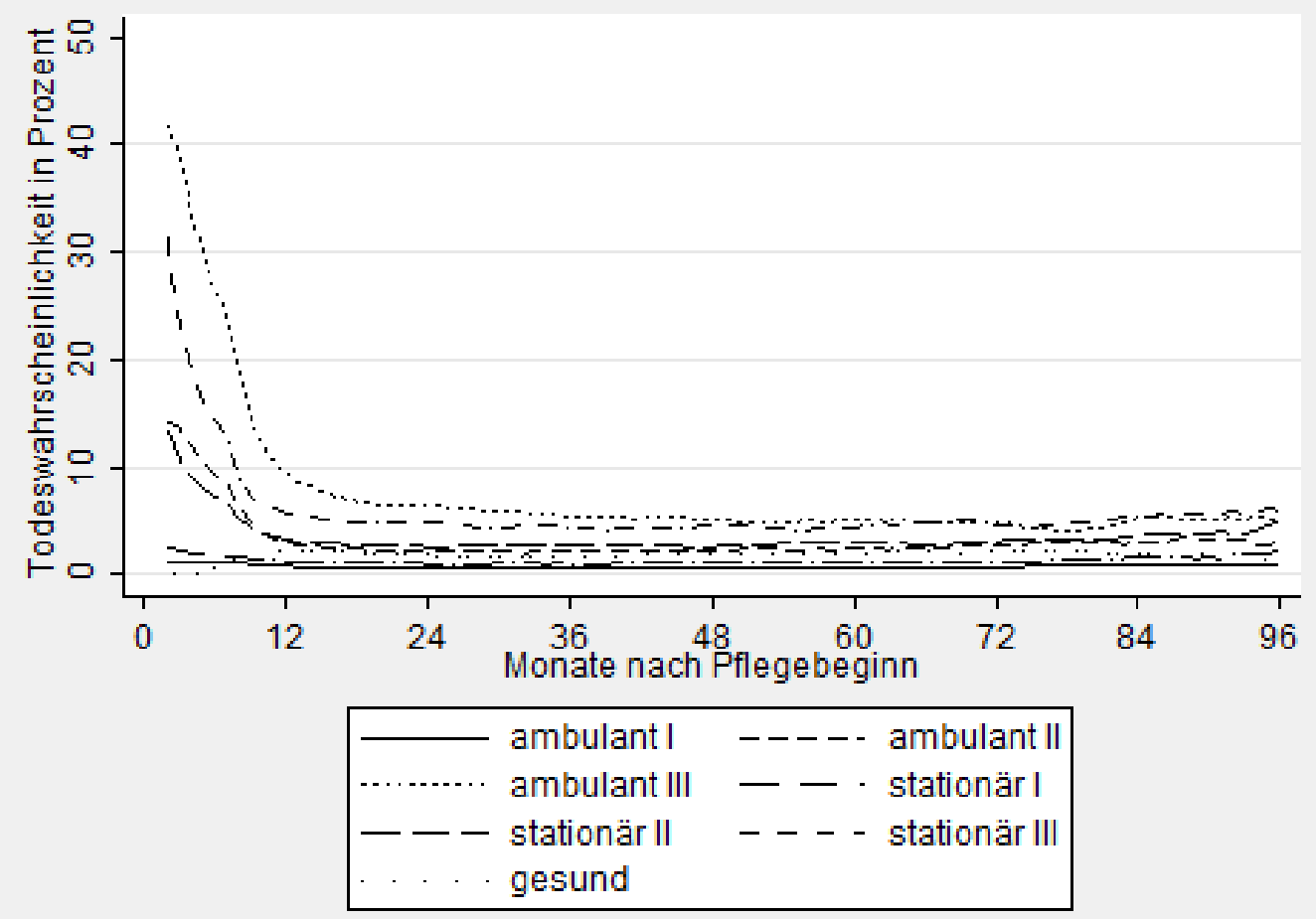

(a) Frauen

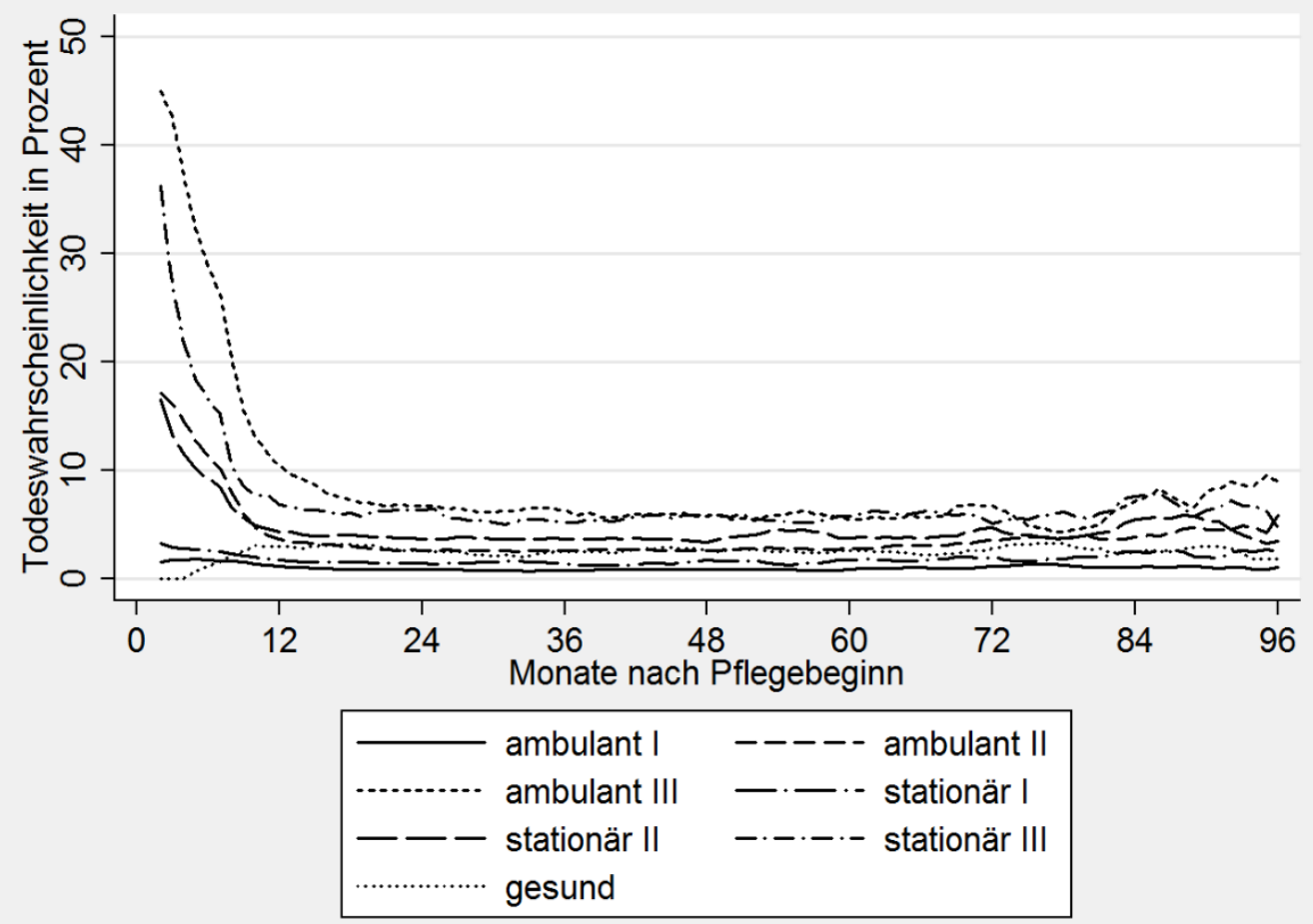

(b) Männer

Quelle: eigene Berechnungen 
Abbildung 9: Differenzierter Pflegeverlauf mit Fortschreibung

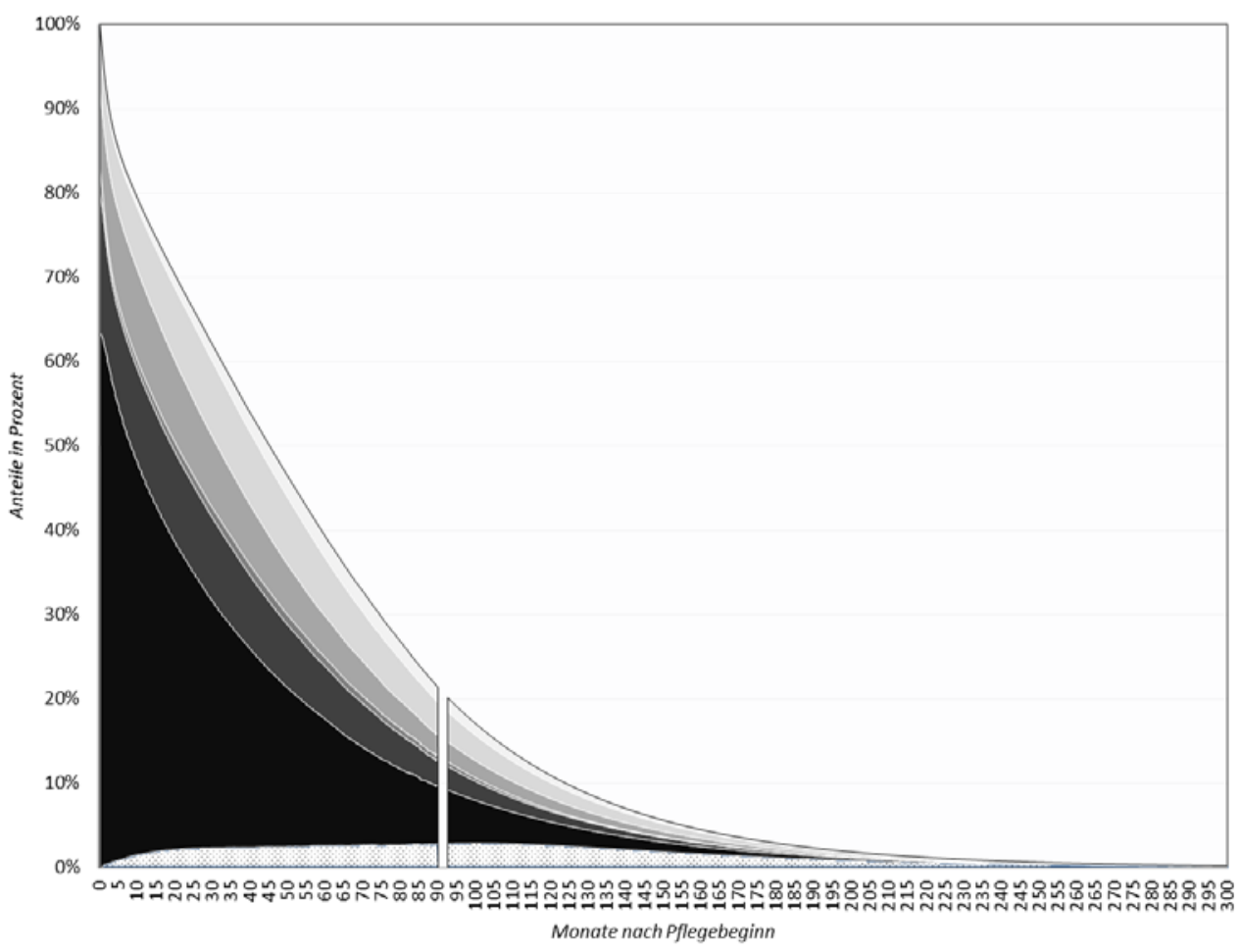

$\square$ tot

III stationär

= II stationär

= I stationär

- III ambulant

- II ambulant

- I ambulant

$\square$ Pflegeunterbrechung

(a) Frauen

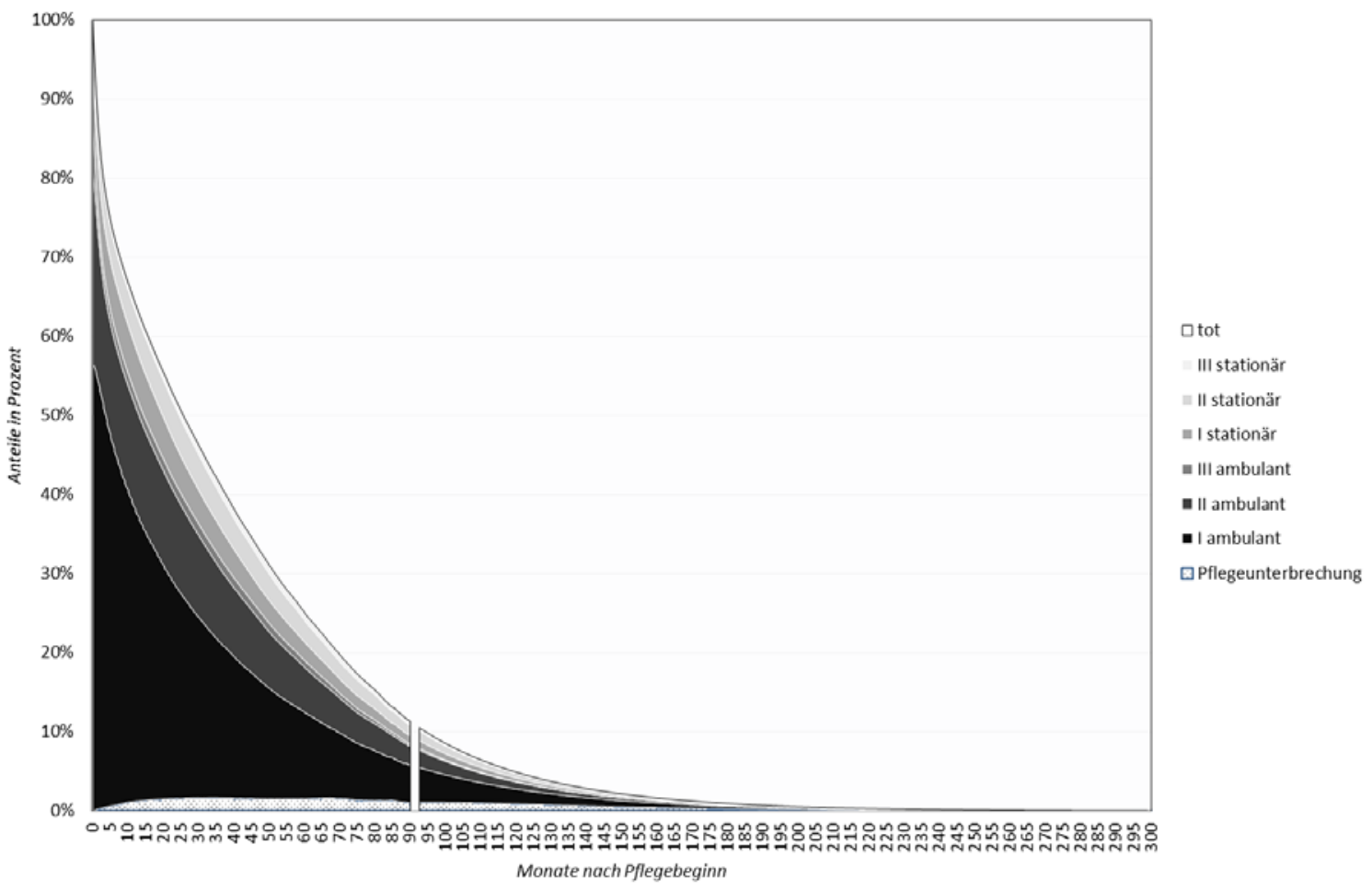

(b) Männer

Quelle: eigene Berechnungen 
Tabelle 10: Deskriptive Statistiken der verwendeten Variablen

\begin{tabular}{|l|ccccc|}
\hline & Beobachtungen & Mittelwert & Standardabweichung & Min & Max \\
\hline Pflegestufe & 6.771 .465 & 1,40756 & 0,5937143 & 1 & 3 \\
geschlecht & 6.771 .465 & 0,7252693 & 0,4463785 & 0 & 1 \\
eintritt & 6.771 .465 & 29,40966 & 21,46612 & 0,033333 & 97,33334 \\
stationär & 6.771 .465 & 0,276086 & 0,4470599 & 0 & 1 \\
tot & 6.771 .465 & 0,479905 & 0,4995961 & 0 & 1 \\
alter & 6.771 .465 & 991,9194 & 83,33966 & 745,5277 & 1293,94 \\
alter2 & 6.771 .465 & 990849,7 & 164404,6 & 555811,6 & 1674282 \\
ttd & 6.771 .465 & 45,17852 & 30,19477 & 0 & 96 \\
ttd2 & 6.771 .465 & 2952,823 & 2920,432 & 0 & 9216 \\
\hline
\end{tabular}

Quelle: eigene Berechnungen 